8.5repared in cooperation with the Calfornia Waterfowl Association, University of California-Davis, and U.S. Fish and Wildlife Service

\title{
Archiving California's Historical Duck Nesting Data
}


Cover: Recently hatched ducklings in a mallard nest at Grizzly Island Wildlife Area, California. Photograph taken by Andrea Mott, U.S. Geological Survey, 2015. 


\section{Archiving California's Historical Duck Nesting Data}

By Joshua T. Ackerman, Mark P. Herzog, Caroline Brady, John M. Eadie, and Greg S. Yarris

Prepared in cooperation with the California Waterfowl Association, University of California-Davis, and U.S. Fish and Wildlife Service

Open-File Report 2015-1131

U.S. Department of the Interior

U.S. Geological Survey 


\section{U.S. Department of the Interior \\ SALLY JEWELL, Secretary}

\section{U.S. Geological Survey \\ Suzette M. Kimball, Acting Director}

U.S. Geological Survey, Reston, Virginia: 2015

For more information on the USGS—-the Federal source for science about the Earth, its natural and living resources, natural hazards, and the environment-visit http://www.usgs.gov or call 1-888-ASK-USGS (1-888-275-8747)

For an overview of USGS information products, including maps, imagery, and publications, visit http://www.usgs.gov/pubprod

Any use of trade, firm, or product names is for descriptive purposes only and does not imply endorsement by the U.S. Government.

Although this information product, for the most part, is in the public domain, it also may contain copyrighted materials as noted in the text. Permission to reproduce copyrighted items must be secured from the copyright owner.

Suggested citation:

Ackerman, J.T., Herzog, M.P., Brady, C., Eadie, J.M., and Yarris, G.S., 2015, Archiving California's historical duck nesting data: U.S. Geological Survey Open-File Report 2015-1131, 26 p., http://dx.doi.org/10.3133/ofr20151131.

ISSN 2331-1258 (online) 


\section{Acknowledgments}

This research was funded by the U.S. Geological Survey Data Rescue Program, Central Valley Joint Venture, and U.S. Geological Survey Ecosystems Mission Area. We thank Ruth Ostroff for project support; Dan Loughman and Bob McLandress for historical knowledge and collaboration; numerous field biologists who collected the data over the past 30 years; and the many technicians who organized, scanned, and entered the data. 
This page left intentionally blank 


\section{Contents}

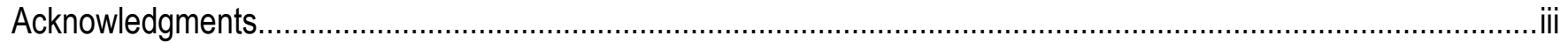

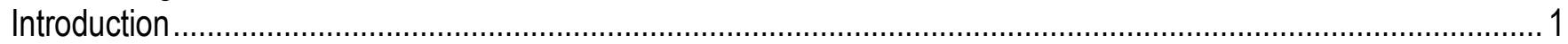

Objectives

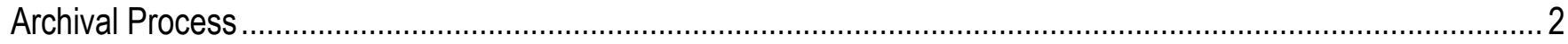

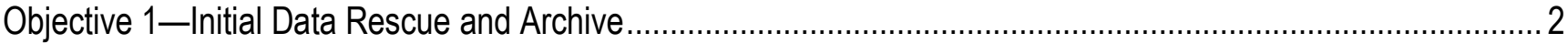

Objective 2-Electronic Archival System .............................................................................................. 2

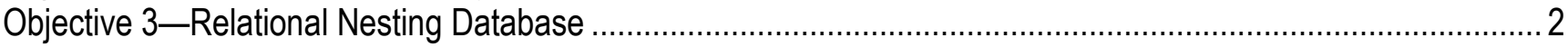

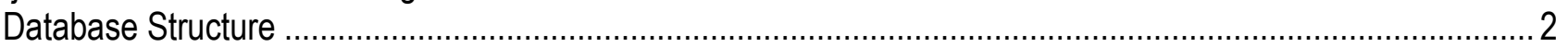

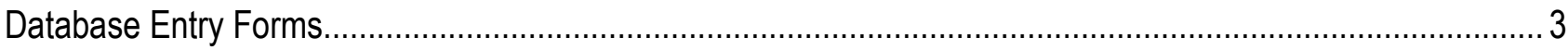

Objective 4-Nest Data Entry .......................................................................................................

Objective 5-California Waterfowl Nest Data Summary ……………………………………......................

Grizzly Island Wildlife Area...............................................................................................................

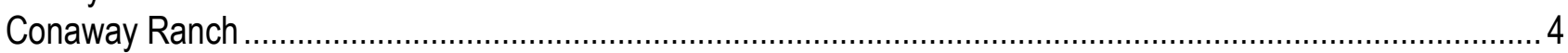

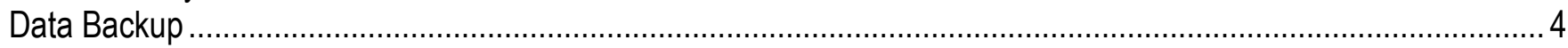

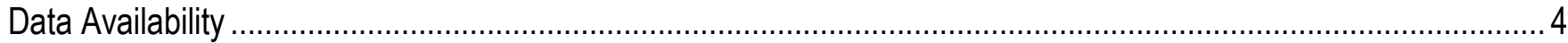

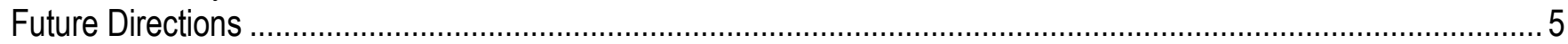

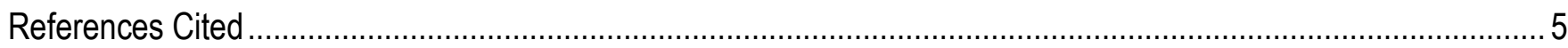

Appendix A. Archival Metadata, Including File Names, Location of Data, Site Names, and Years ......................... 14

Appendix B. Summary of All Duck Nest Data Collected by Study Region, Field, and Year,

California, 1985-2014.

\section{Figures}

Figure 1. Nest database structure, including relationships between data entry tables

Figure 2. Nest database entry login screen for the California Avian Nesting Database Entry Archive (CANDE Archive), which contains the data for this current project, Archiving California's Historical Duck Nesting Data........ 7

Figure 3. Example of main nest database entry page, showing entry of visit number 1 for nest number 2 in field $13 E$ at Grizzly Island Wildlife Area in 2008

Figure 4. Example of nest database entry page for the partial nest depredation subform .................................... 9

Figure 5. Example of nest database entry page for the nest-captured hen data subform .................................... 9

Figure 6. Summary of all waterfowl and associated species nest data, California, 1985-2014 ............................ 10

Figure 7. Summary of all waterfowl and associated species nest data by study region and nesting bird species, California, 1985-2014

Figure 8. Summary of all waterfowl and associated species nest data by study region, California, 1985-2014 .... 12 Figure 9. Summary of all waterfowl and associated species nest data collected by field at Grizzly Island Wildlife Area, California, 1985-2014 
This page left intentionally blank 


\title{
Archiving California's Historical Duck Nesting Data
}

\author{
By Joshua T. Ackerman¹, Mark P. Herzog1ㅜ Caroline Brady², John M. Eadie³, and Greg S. Yarris4
}

\section{Introduction}

The U.S. Geological Survey (USGS), in partnership with the California Waterfowl Association (CWA) and other organizations, have compiled large datasets on the nesting ecology and management of dabbling ducks and associated upland nesting birds (Northern Harriers [Circus cyaneus], Short-eared Owls [Asio flammeus], Ring-necked Pheasants [Phasianus colchicus], and American Bitterns [Botaurus lentiginosus]) throughout California on Federal Refuges, State Wildlife Areas, and private lands, some participating in State and Federal habitat programs. These datasets encompass several long-term monitoring programs at multiple sites throughout California, and include data from more than 26,000 nests and span nearly 30 years.

These historical datasets represent some of the longest term datasets on nesting ducks in North America, if not the world. They are extremely valuable for ongoing waterfowl management and habitat conservation efforts in California, as well as throughout the world. However, without organization and electronic access, these data are an untapped resource and are not being used to the full extent possible. Prior to this project, these datasets were scattered among various agencies and organizations, and original paper nest cards were being stored in cardboard boxes in attics and storage containers that were not suitable for long-term archival storage. In addition, most of these data had not been entered into a computerized database and thus were at high risk for permanent data loss.

To protect this irreplaceable dataset, we submitted a series of proposals to obtain funds to complete this data archival project over the past 5 years. The Central Valley Joint Venture, USGS Data Rescue Program, and USGS Ecosystems Mission Area funded this data archival project. In addition, we leveraged other USGS projects on nesting shorebirds, songbirds, and seabirds to use further resources to more fully develop the nest database structure for use on nesting waterfowl. Specifically, this large dataset on ducks was archived by USGS, but the dataset is owned and managed by a consortium of organizations. Therefore, any access and use of this data must occur through the principal investigators, who contributed data and resources to this archival project, as detailed in section, "Data Availability."

With the conclusion of this project, most duck nest data have been entered, but all nest-captured hen data and other breeding waterfowl data that were outside the scope of this project have still not been entered and electronically archived. Maintaining an up-to-date archive will require additional resources to archive and enter the new duck nest data each year in an iterative process. Further, data proofing should be conducted whenever possible, and also should be considered an iterative process as there was sometimes missing data that could not be filled in without more direct knowledge of specific projects. Despite these disclaimers, this duck data archive represents a massive and useful dataset to inform future research and management questions.

\footnotetext{
${ }^{1}$ U.S. Geological Survey.

${ }^{2}$ California Waterfowl Association.

${ }^{3}$ University of California, Davis.

${ }^{4}$ Central Valley Joint Venture.
} 


\section{Objectives}

The objectives of this project were to:

1. Work with various organizations to locate, obtain, and organize all data on nesting ducks in California.

2. Build an electronic archival system for long-term storage, and electronically scan all nesting data.

3. Build an enterprise-level nesting database in a relational database management system to enter and store these data with a Microsoft ${ }^{\circledR}$ Access user interface.

4. Enter the archived data into the nesting database.

5. Summarize available waterfowl nesting data in California.

\section{Archival Process}

\section{Objective 1-Initial Data Rescue and Archive}

We searched for all available waterfowl nesting datasets in California, including the California Waterfowl Association, USGS, and University of California-Davis (UC Davis) offices and field stations. Much of the data were found in storage rooms, garages, and attics of buildings and stored in lightweight cardboard boxes. All data and documents were collected, consolidated into a single location, and reviewed and organized according to project and data type. Currently, all original nesting files have been organized and filed in metal file cabinets at the USGS Dixon Field Station.

\section{Objective 2-Electronic Archival System}

After data were organized, we electronically scanned all waterfowl nest data and saved as Adobe ${ }^{\circledR}$ Acrobat ${ }^{\circledR}$ pdf files. During the process of organizing and scanning the data, we developed a spreadsheet inventory for all existing data (appendix A). The inventory documents the data type, physical file location, electronic file location, data entry status, and additional metadata for each piece of data.

\section{Objective 3-Relational Nesting Database}

\section{Database Structure}

The primary data of interest were nesting waterfowl data. To make these nesting data more accessible, we developed a relational database to store nest data (fig. 1). Briefly, this database collects all nest information including specific information on nest visits, nest attributes, spatial location of nest, depredation events, individual eggs, and vegetation at the nest. In addition to nest data, the database also stores data on female ducks that were captured on the nest and banded (although these data have not been entered into the database). The properties and structure of the database allows for rigorous proofing routines to be performed for ensuring the integrity of the data. Given the structure of the relational database, these tables can be queried and merged together by a trained user to develop complex datasets for statistical analysis. 


\section{Database Entry Forms}

The data entry program provides a structured environment where the data can be entered and accessed simultaneously by multiple users, facilitating rapid entry if staff and resources are available (fig. 2). Within the nest database, structured forms and subforms (figs. 3-5) enforce quality control and reduce the number of data entry errors. The database environment also allows for easy data revision and quality control. To avoid errors, access to the nest database is restricted to approved and trained users only, and roles define whether users can enter, edit, or only view data. Approved users are trained and provided an instruction manual that fully describes the components of the nest database as well as detailed standard procedures for data entry and specific data entry activities, such as nest fate determination.

\section{Objective 4-Nest Data Entry}

Over a period of 4 years, most of the waterfowl nest data were entered into the nest database. We have entered nest data from 1985 to 2014, which includes more than 26,500 nests from 118 site-years. More nest data are still available for entry but entering these additional nest data is beyond the scope of this project. Original nest data also should be continually proofed as resources become available, as part of an iterative process of data management. Sample sizes of nests monitored by study site, cooperators involved in the data collection, number of years sampled, and availability of associated metadata are shown in figure 6.

\section{Objective 5-California Waterfowl Nest Data Summary}

From 1985 to 2014, a total of 26,578 duck and associated upland nesting bird nests were monitored (fig. 6). The species of nesting bird by study region is shown in figure 7. The most duck nest monitoring data are from the Grizzly Island Wildlife Area (17,685 nests), Sacramento National Wildlife Area (1,923 nests), Conaway Ranch (1,374 nests), Mendota Wildlife Area (1,258 nests), Northeastern California (1,063 nests), and Gray Lodge Wildlife Area (492 nests). Although the subsites are more dispersed, a substantial amount of nest data comes from various Sacramento Valley sites (such as Conservation Reserve Enhancement Program [CREP] sites [579 nests] and Fendt [350 nests]). In particular, Grizzly Island Wildlife Area (26 years) and Conaway Ranch (13 years) have been the primary study sites for nesting ducks in California, and have the longest time series of nest data (fig. 8). No other study site has more than 8 years of nest data, but other moderately sized datasets include Gray Lodge (8 years), Thermalito Afterbay (6 years), Mendota (5 years), Sacramento Valley (5 years), CREP Colusa County (5 years), CREP Yolo County ( 5 years), Fendt (5 years), Kalfsbeek (5 years), Beale Air Force Base (4 years), Los Banos (4 years), Delevan National Wildlife Refuge (3 years), Palm Tract (3 years), and Yolo Bypass (3 years; fig. 8). The number of nests monitored by year and subsite for each study region is shown in appendix $\mathrm{B}$. 


\section{Grizzly Island Wildlife Area}

By far, the most duck nest data have been collected at the Grizzly Island Wildlife Area study site, which has been a long-term collaboration between CWA, USGS, and UC Davis. CWA started monitoring this site in 1985 (McLandress and Yarris, oral commun., 2015), and UC Davis (Ackerman and Eadie, authors unpublished) began collaborating in 1997. Since then, CWA, USGS, and UC Davis have tried to maintain this annual nesting program. This dataset has been used to generate several scientific publications describing duck nesting ecology (Mclandress and others, 1996; Ackerman and others, 2003a, 2014), molt migration movements (Yarris and others, 1994), density dependent nest survival (Ackerman and others, 2004; Ringelman and others, 2012, 2013), influence of alternate prey on duck nest survival (Ackerman, 2002), and parental investment behaviors of nesting ducks (Ackerman and Eadie, 2003; Ackerman and others, 2003b).

Grizzly Island Wildlife Area has been monitored almost continuously from 1985 to 2014, except for 2005-07 and 2014 (fig. 8). The most nests monitored at Grizzly Island Wildlife Area was in 1996 (1,259 nests) and the fewest nests in 2004 (178 nests; fig. 8). In general, fewer nests have been found at Grizzly Island Wildlife Area in recent years (after 2001) primarily because of substantially reduced nest monitoring efforts (namely, fewer fields searched each year) because of limited resources. There was a substantial increase in nests monitored at Grizzly Island Wildlife Area from 2010 to 2012 during the Wildlife Conservation Board grant obtained by CWA and USGS to improve the habitat in the nesting fields, which provided additional resources for nest monitoring. The number of nests monitored by year within each of the nesting fields at Grizzly Island Wildlife Area are shown in figure 9. This extensive nesting database has been useful in guiding habitat management and restoration plans in Suisun Marsh (Ackerman and others, 2014).

\section{Conaway Ranch}

One of the more successful partnerships with the private sector has been the collaboration with Conaway Ranch. CWA has monitored duck nesting every year from 1995 to 2007 at Conaway Ranch, finding between 52 and 187 nests each year.

\section{Data Backup}

The nest database and the scanned pdf files of the raw data are stored on a USGS network server in Dixon, California that is regularly backed up to hard drives in Sacramento, California. A copy of the data also is stored on an external hard drive and regularly updated.

\section{Data Availability}

Waterfowl nesting data collected in California are the result of a number of organizations and cooperators dedicated to understanding and guiding the management of breeding waterfowl in California and represent tens of millions of dollars spent on research and data management over the past 30 years. Those interested in using these data should contact the curators of Archiving California's Historical Duck Nesting Data at USGS (Josh Ackerman and Mark Herzog) with the proposed collaboration, data request, and summary of the proposed products. The proposed collaboration will then be circulated among cooperators for further discussion about collaboration, individual responsibilities, and co-authorship on developed products. 


\section{Future Directions}

As part of this archival process, we also identified additional breeding waterfowl datasets that were outside the scope and funding of the current project. The breeding waterfowl data that have not been entered into the archival system includes recent nest data, several types of metadata (maps, nest coordinates produced on maps, extra vegetation surveys, brood surveys, predator surveys, egg size measurements, and other metadata), summer bait-trapped banded ducks (body condition and banding data), and nest-captured hen database (approximately 3,000 individual hens captured on nests from 1985 to 2014). These additional breeding waterfowl data should be entered as resources become available, especially the valuable nest-captured hen database. Additionally, this dataset will require continued maintenance, and upkeep of entry of new nest data.

\section{References Cited}

Ackerman, J.T., 2002, Of mice and mallards - positive indirect effects of coexisting prey on waterfowl nest success: Oikos, v. 99, no. 3, p. 469-480.

Ackerman, J.T., Blackmer, A.L., and Eadie, J.M., 2004, Is predation on waterfowl nests density dependent? - tests at three spatial scales: Oikos, v. 107, no. 1, p. 128-140.

Ackerman, J.T., and Eadie, J.M., 2003, Current versus future reproduction: an experimental test of parental investment decisions using nest desertion by mallards (Anas platyrhynchos): Behavioral Ecology and Sociobiology, v. 54, no. 3, p. 264-273.

Ackerman, J.T., Eadie, J.M., Loughman, D.L., Yarris, G.S., and McLandress, M.R., 2003a, The influence of partial clutch depredation on duckling production: Journal of Wildlife Management, v. 67, no. 3, p. 576-587.

Ackerman, J.T., Eadie, J.M., Yarris, G.S., Loughman, D.L., and McLandress, M.R., 2003b, Cues for investment: nest desertion in response to partial clutch depredation in dabbling ducks: Animal Behaviour, v. 66, no. 5, p. 871-883.

Ackerman, J.T., Herzog, M.P., Yarris, G.S., Casazza, M.L., Burns, E.W., and Eadie, J.M., 2014, Waterfowl ecology and management, in Moyle, P.B., Manfree, A., and Fiedler, P.L., eds., Suisun Marsh — ecological history and possible futures : Berkeley, California, University of California Press, p. 103-132.

Mclandress, M.R., Yarris, G.S., Perkins, A.E.H., Connelly, D.P., and Raveling, D.G., 1996, Nesting biology of mallards in California: Journal of Wildlife Management, v. 60, no. 1, p. 94-107.

Ringelman, K.M., Eadie, J.M., and Ackerman, J.T., 2012, Density-dependent nest predation in waterfowl - the relative importance of nest density versus nest dispersion: Oecologia, v. 169, no. 3, p. 695-702.

Ringelman, K.M., Eadie, J.M., and Ackerman, J.T., 2013, Adaptive nest clustering and densitydependent nest survival in dabbling ducks: Oikos, v. 123, no. 2, p. 239-247.

Yarris, G., McLandress, M., and Perkins, A., 1994, Molt migration of postbreeding female Mallards from Suisun Marsh, California: Condor, v. 96, p. 36-45. 


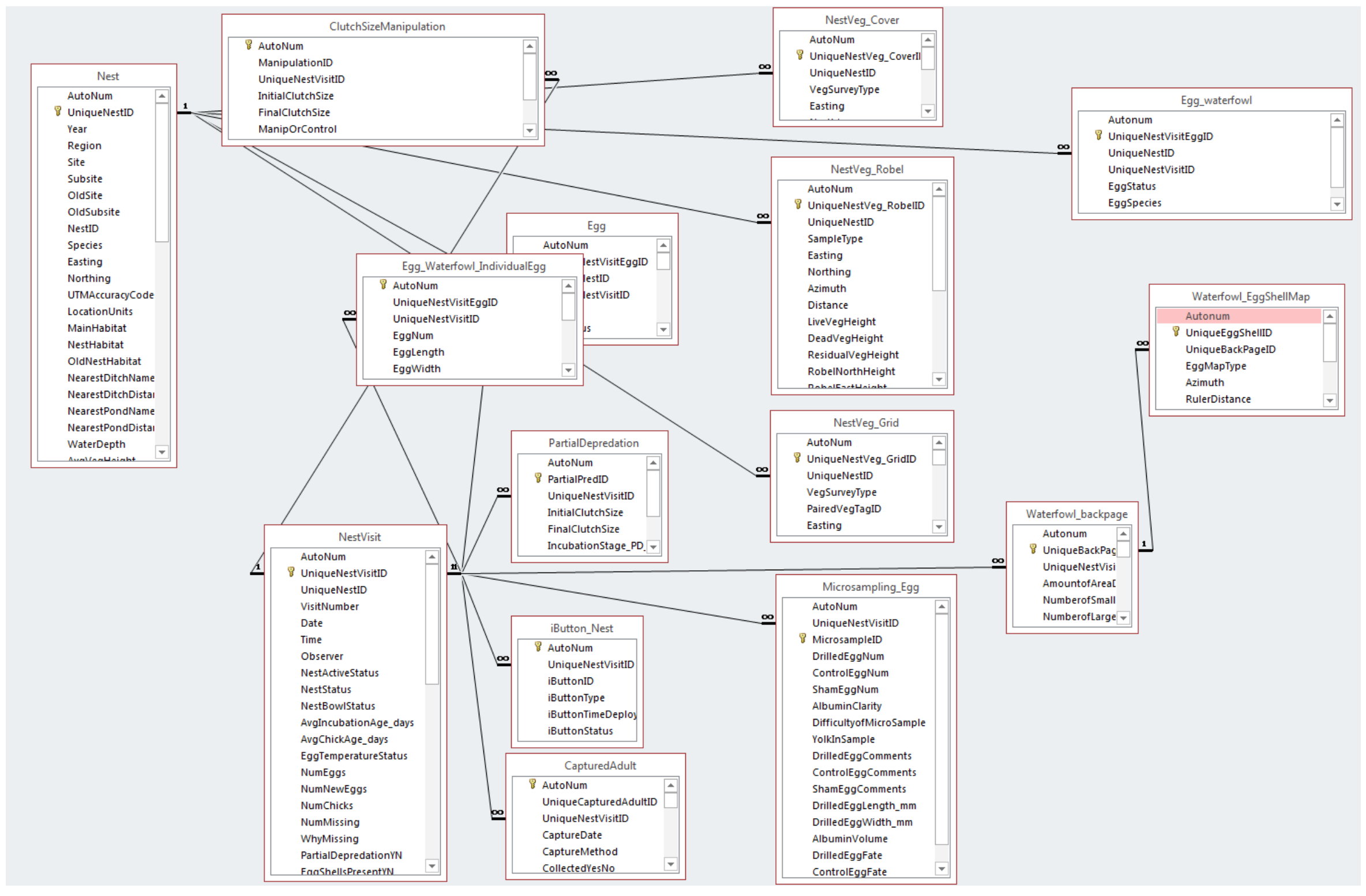

Figure 1. Nest database structure, including relationships between data entry tables. 

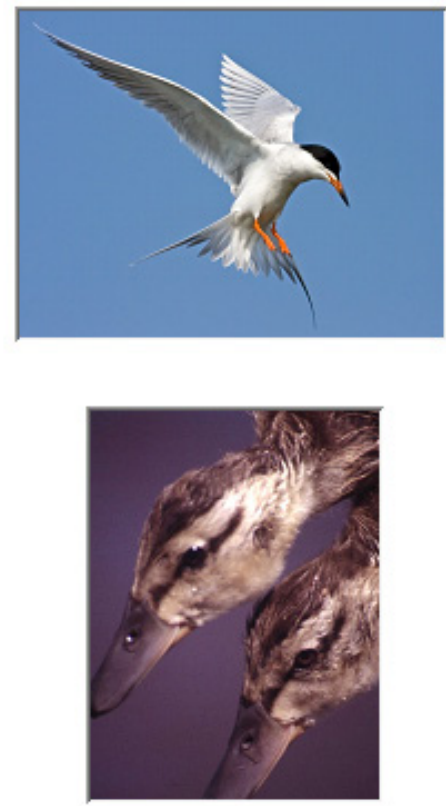

TIP: When PROJECT= "Waterfowl": NestID should be site nestnumber. For other birds use site_species_nestnumber

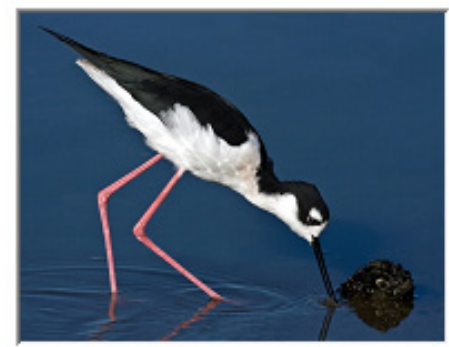

Form Version: 09.30.2014

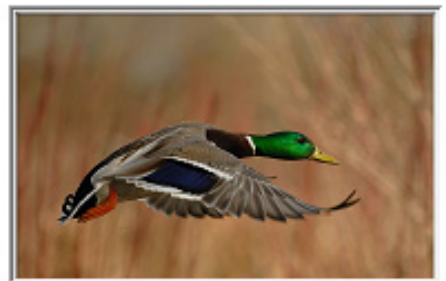

Avian Nest Data Entry
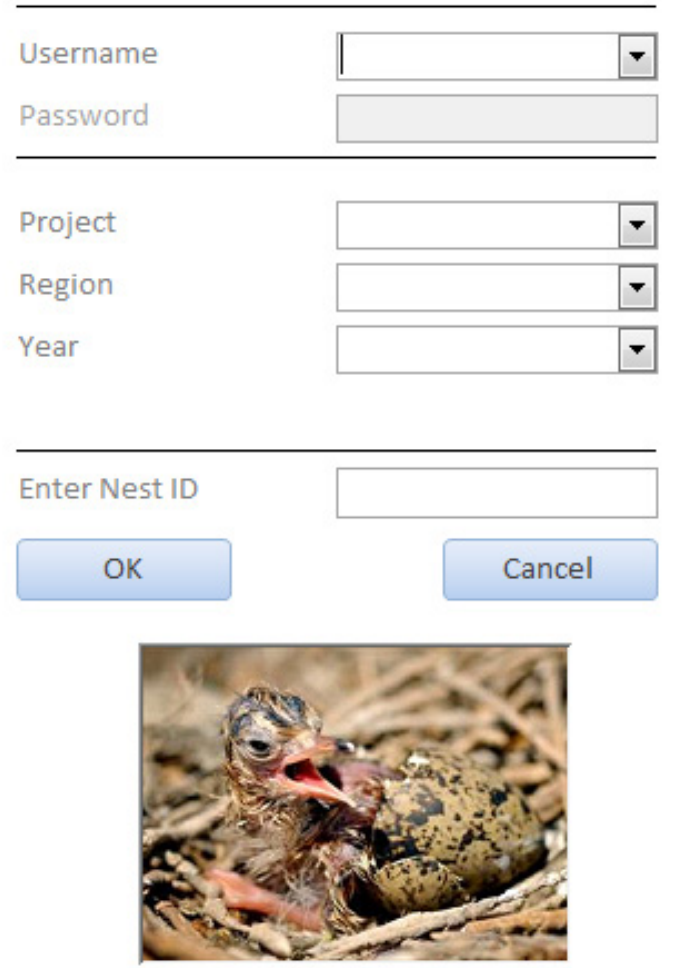

Supplemental Data Version: 9.30.2014
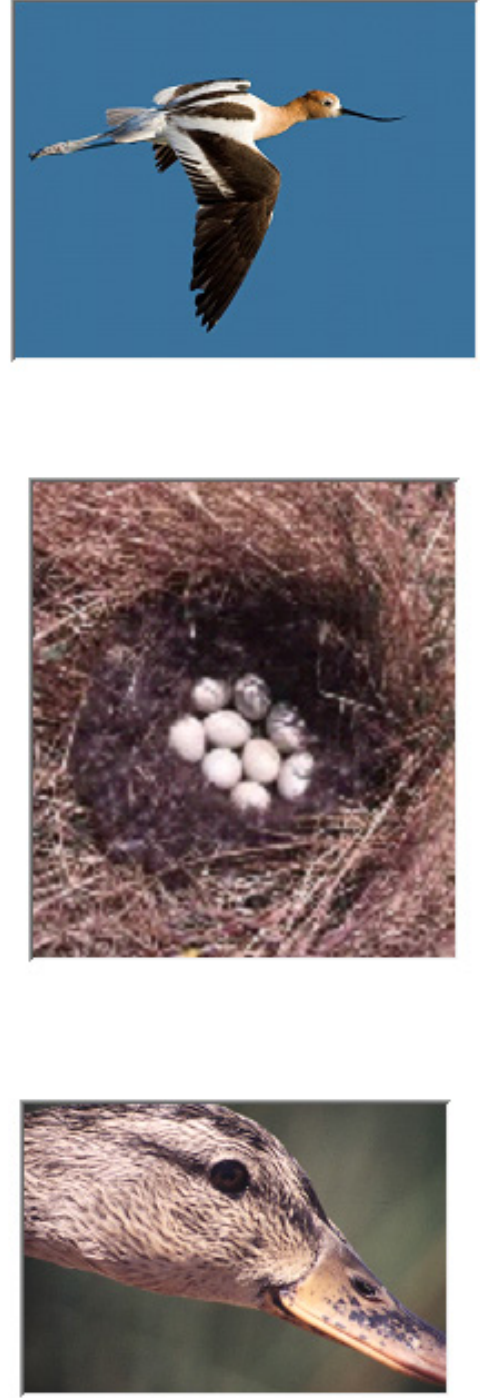

Database Version: 09.30.2014

Figure 2. Nest database entry login screen for the California Avian Nesting Database Entry Archive (CANDE Archive), which contains the data for this current project, Archiving California's Historical Duck Nesting Data. 

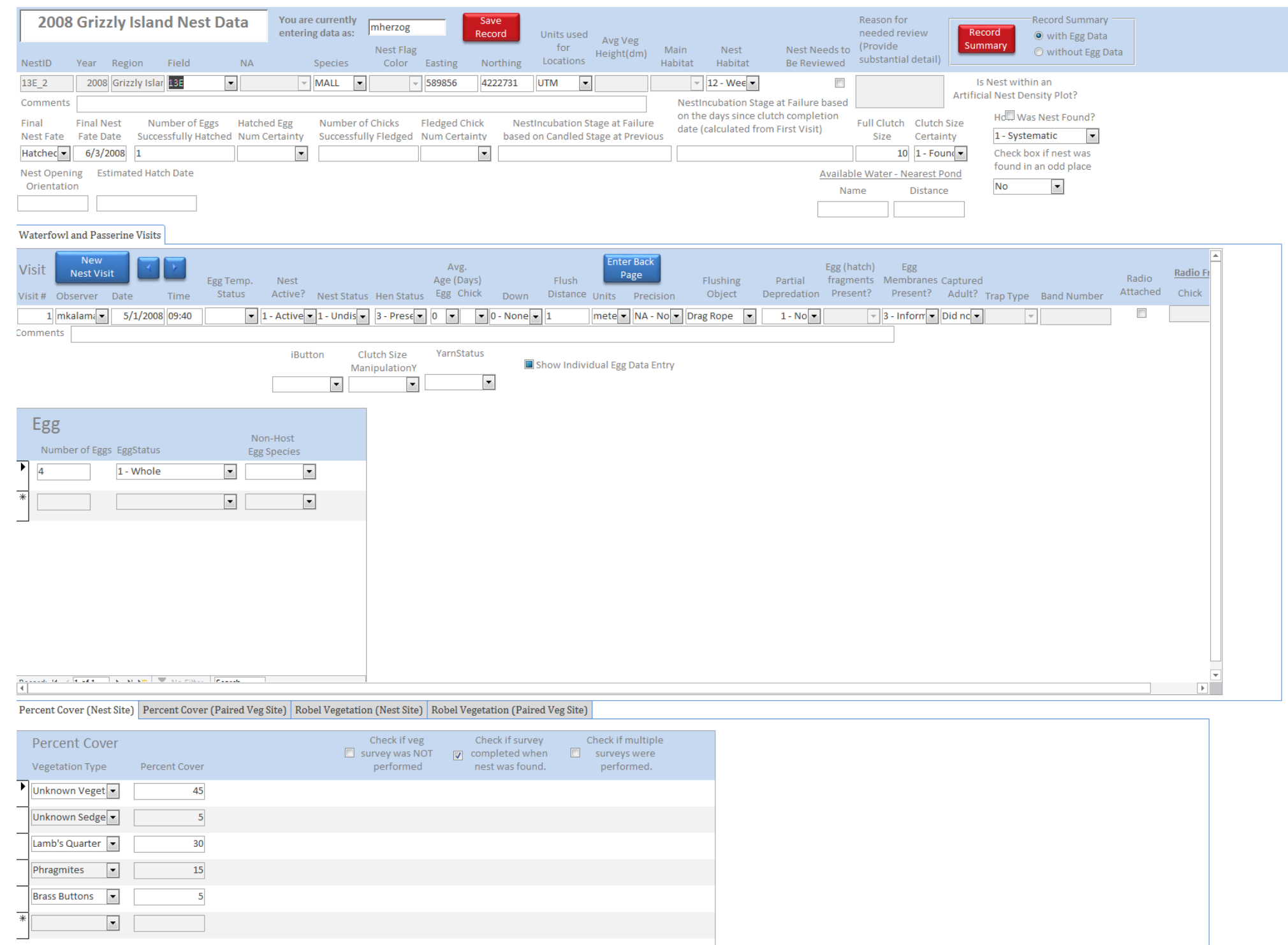

Figure 3. Example of main nest database entry page, showing entry of visit number 1 for nest number 2 in field 13E at Grizzly Island Wildlife Area in 2008. 


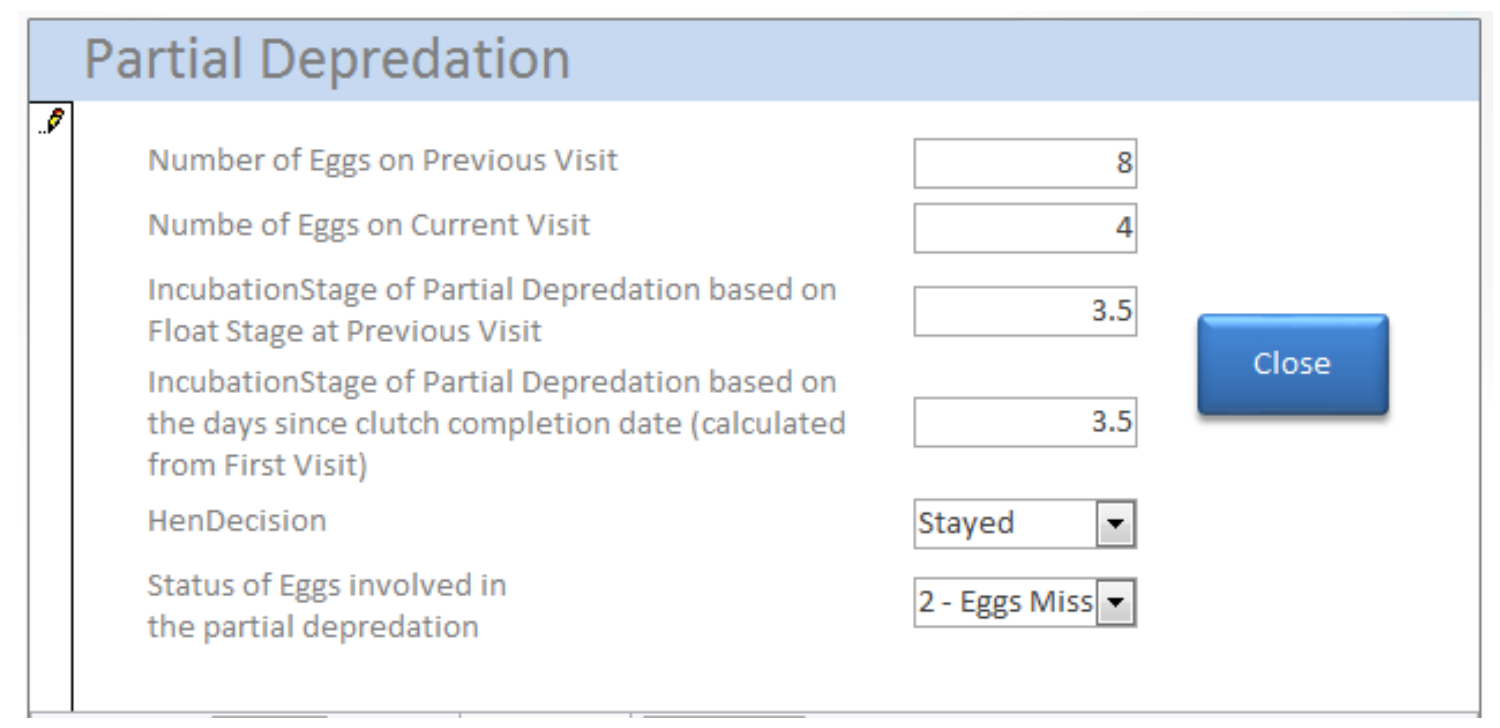

Figure 4. Example of nest database entry page for the partial nest depredation subform.

\begin{tabular}{l} 
Capture Record \\
Capture Date Capture Method Time Trap was set Capture Time Release Time Processing Group \\
Species Sex of Bird On Nest At Set Time Sex of Captured Bird Band Number \\
Age Fulmen Length Short Tarsus Length Flattened Wing Length BirdandBagMass BagMass \\
\hline ASY Female
\end{tabular}

Figure 5. Example of nest database entry page for the nest-captured hen data subform. Although the nest-captured hen subform was developed, the hen data has not been entered as it was beyond the scope of this project. 


\begin{tabular}{|c|c|c|c|c|c|c|c|c|}
\hline Study Site & Landowner & Cooperators $^{1}$ & Study Period & $\begin{array}{l}\text { Number } \\
\text { of Years }\end{array}$ & $\begin{array}{l}\text { Number } \\
\text { of Nests }\end{array}$ & $\begin{array}{l}\text { Bird Banding } \\
\text { Data: Nest } \\
\text { Trapped } \\
\text { Hens } \\
\end{array}$ & $\begin{array}{l}\text { Bird } \\
\text { Banding } \\
\text { Data: Bait } \\
\text { Trapped } \\
\end{array}$ & Other Data \\
\hline Grizzly Island WA & State & USGS/CWA/UC Davis/CDFW/WCB & 1985-2004;2008-2013 & 26 & 17,685 & Yes & Yes & Brood, predator, and vegetation surveys \\
\hline Conaway Ranch & Private & Private Landowner/CWA Collaboration & 1995-2007 & 13 & 1,374 & Yes & Yes & Vegetation surveys \\
\hline Butte Valley WA & State & CWA/CDFW & 1988 & 1 & 45 & Yes & Yes & Vegetation surveys \\
\hline Gray Lodge WA & State & CWA/CDFW & 1986-2001 & 8 & 492 & Yes & Yes & Brood, predator, and vegetation surveys \\
\hline $\begin{array}{l}\text { Honey Lake/Ash Creek } \\
\text { WA }\end{array}$ & State & CWA/CDFW & 1987-1989 & 3 & 1063 & Yes & Yes & Brood, predator, and vegetation surveys \\
\hline Los Banos WA & State & CWA/CDFW & 1989-1990; 1992-1993 & 4 & 189 & No & No & None \\
\hline Mendota WA & State & CWA/CDFW & 1987-1989 & 5 & 1258 & Yes & Yes & Brood, predator, and vegetation surveys \\
\hline Napa-Sonoma Marsh WA & State & CWA/CDFW & 1994 & 1 & 13 & No & No & Vegetation surveys \\
\hline North Grasslands WA & State & CWA/CDFW & 1992-1993 & 2 & 84 & No & No & Hunting results \\
\hline Thermalito Afterbay & State & CSU San Jose/CDFW/CWA/DWR Waterfowl Monitoring Program & 1987-1994 & 6 & 403 & No & No & Vegetation surveys \\
\hline Yolo Bypass & State & CWA/CDFW & 1986; 1988; 2008 & 3 & 91 & No & No & None \\
\hline Beale Air Force Base & Federal & USDoD/CWA Waterfowl and Habitat Evaluation Project & 1994-1997 & 4 & 101 & No & No & Vegetation surveys \\
\hline Colusa NWR & Federal & CWA/USFWS & 1986-1988 & 3 & 72 & No & No & Breeding pair and vegetation surveys \\
\hline Delevan NWR & Federal & CWA/USFWS & 1986-1988 & 4 & 276 & No & No & None \\
\hline Sacramento NWR & Federal & CWA/USFWS & 1986-1991 & 5 & 1923 & Yes & Yes & Brood, predator, and vegetation surveys \\
\hline Tule Lake NWR & Federal & CWA/USFWS & 1988 & 1 & 122 & Yes & No & Vegetation surveys \\
\hline $\begin{array}{l}\text { Davis Water Treatment } \\
\text { Ponds }\end{array}$ & Private & CWA/City of Davis & 1992 & 1 & 26 & No & No & Vegetation surveys \\
\hline Denverton & Private & CWA & 2013 & 1 & 2 & No & No & None \\
\hline Fendt & Private & CWA/Private Landowner & 2002-2007 & 5 & 350 & No & No & Vegetation surveys \\
\hline Garibaldi & Private & CWA/Private Landowner (and now CDFW property at GIWA) & 1988 & 1 & 5 & No & No & None \\
\hline Kalfsbeek & Private & CWA/Private Landowner & 2002-2006 & 5 & 116 & No & No & None \\
\hline Mandeville Island & Private & CWA/Private Landowner & 1988; 1991; 2004 & 3 & 122 & No & Yes & Vegetation surveys \\
\hline $\begin{array}{l}\text { Mountain Meadows } \\
\text { Reservoir }\end{array}$ & Private & CWA/Private Landowner/WCB Upland Enhancement Evaluation & 1997; 1999; 2001 & 3 & 11 & No & No & Vegetation surveys \\
\hline Palm Tract & Private & CWA/Private Landowner/CDFW/COTP Mitigation Evaluation & 1996-1998 & 3 & 88 & No & No & Vegetation surveys \\
\hline Riley Farms & Private & CWA/Private Landowner (winter wheat next to Gray Lodge WA) & 1986 & 1 & 25 & No & No & None \\
\hline $\begin{array}{l}\text { Sacramento Valley Ag. } \\
\text { Habitats }\end{array}$ & Private & USDA-CWA Conservation Reserve Enhancement Program & 2002-2006 & 5 & 579 & Yes & Yes & Breeding pair and vegetation surveys \\
\hline Winters & Private & CWA/Private Landowner (Rominger or John Anderson/Hedgerow Farms) & 1988 & 1 & 5 & No & No & None \\
\hline & & & \multicolumn{3}{|c|}{ TOTAL $=118$ site-years \& 26,578 nests } & & & \\
\hline
\end{tabular}

Figure 6. Summary of all waterfowl and associated species nest data, California, 1985-2014. Cooperators include U.S. Geological Survey (USGS), California Department of Fish and Wildlife (CDFW), California Waterfowl Association (CWA), Wildlife Conservation Board (WCB), University of California, Davis (UC Davis), U.S. Fish and Wildlife Service (USFWS), U.S. Department of Agriculture (USDA), California State University (CSU), Department of Water Resources (DWR), and U.S. Department of Defense (DOD). 


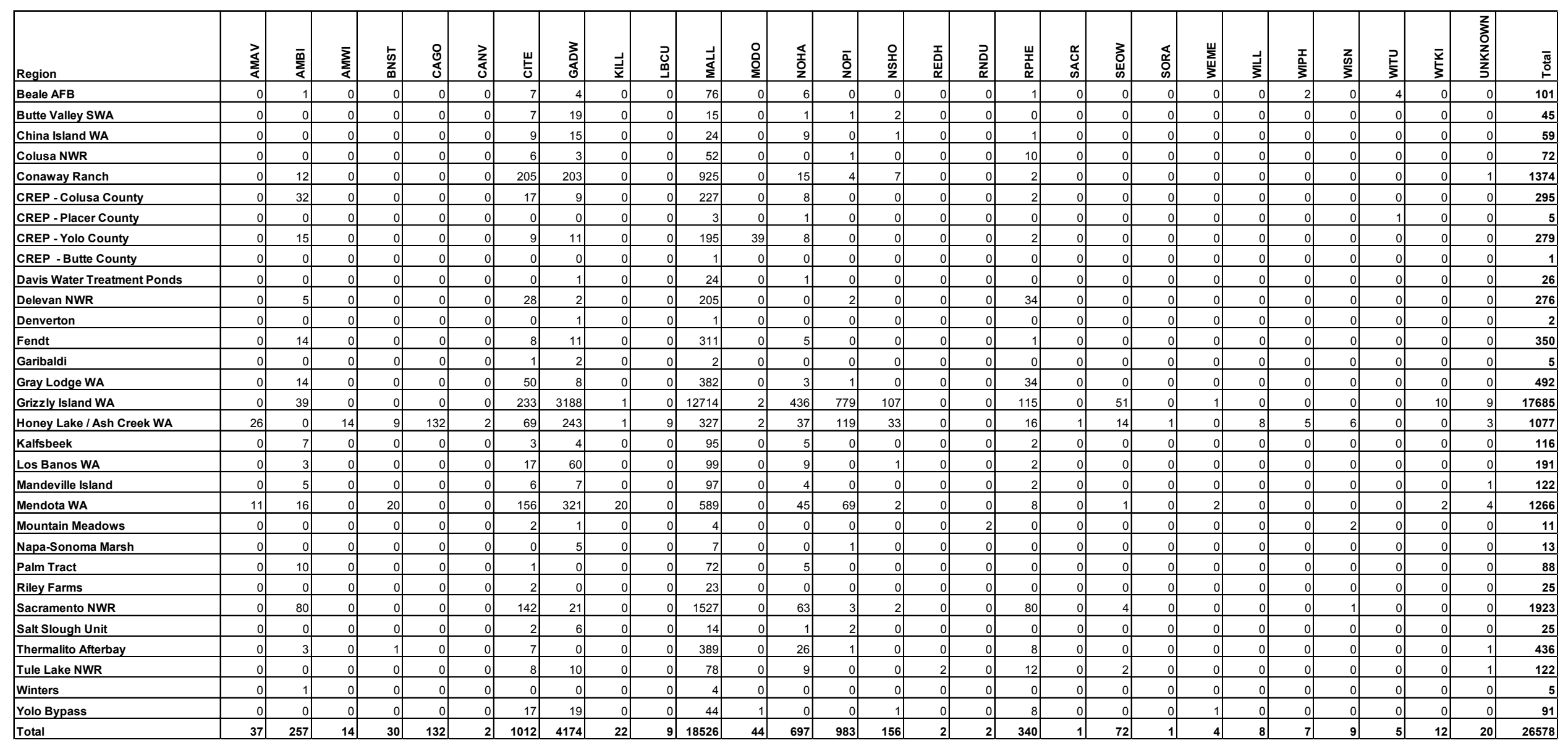

Figure 7. Summary of all waterfowl and associated species nest data by study region and nesting bird species, California, 1985-2014. Species are American Avocet (AMAV), American Bittern (AMBI), Black-necked Stilt (BNST), Canada Goose (CAGO), Canvasback (CANV), Cinnamon Teal (CITE), Gadwall (GADW), Killdeer (KILL), Long-billed Curlew (LBCU), Mallard (MALL), Mourning Dove (MODO), Northern Harrier (NOHA), Northern Pintail (NOPI), Northern Shoveler (NSHO),

Redhead Duck (REDH), Ring-necked Duck (RNDU), Ring-necked Pheasant (RPHE), Sandhill Crane (SACR), Short-eared Owl (SEOW), Sora (SORA), Western Meadowlark (WEME), Willet (WILL), Wilson's Phalarope (WIPH), Wilson's Snipe (WISN), Wild Turkey (WITU), and White-tailed Kite (WTKI). 


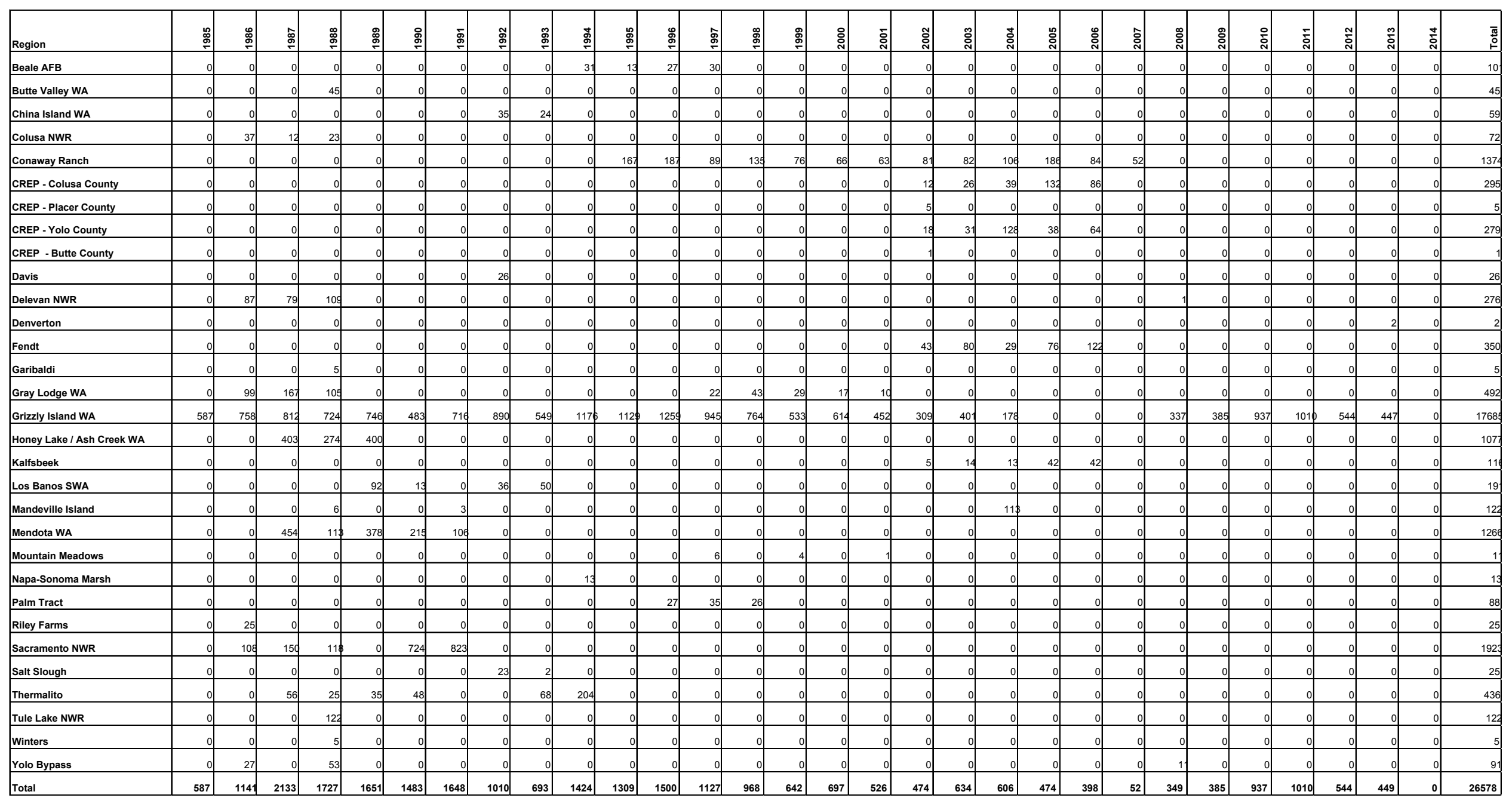

Figure 8. Summary of all waterfowl and associated species nest data by study region, California, 1985-2014. 


\begin{tabular}{|c|c|c|c|c|c|c|c|c|c|c|c|c|c|c|c|c|c|c|c|}
\hline Site & 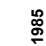 & 怘 & 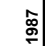 & $\begin{array}{c}\infty \\
\stackrel{\infty}{\circ}\end{array}$ & $\begin{array}{l}\stackrel{\circ}{\circ} \\
\stackrel{\circ}{\sigma}\end{array}$ & ঃ & ఏ్ & 胥 & ฏ & 㗊 & 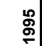 & ঃ & ఏ. & 足 & ঃ & : & ప్స & ธ్రి & స్ \\
\hline 1 & 0 & 0 & 0 & 0 & 0 & 0 & 0 & 0 & 0 & 0 & 0 & 0 & 0 & 0 & 0 & 0 & 0 & 0 & 0 \\
\hline 10 & 0 & 1. & 0 & 0 & 0 & 0 & 0 & 0 & 0 & 0 & 0 & 0 & 0 & 0 & 0 & 0 & 0 & 0 & 0 \\
\hline $10 \mathrm{C}$ & 0 & ol & 0 & 0 & 0 & 0 & 0 & 0 & 0 & 0 & 0 & 0 & 0 & 0 & 0 & 0 & 0 & 0 & 0 \\
\hline $10 \mathrm{D}$ & - & & 0 & 0 & 0 & 0 & 0 & 0 & 0 & 0 & 0 & 0 & 0 & 0 & 0 & 0 & 0 & 0 & 0 \\
\hline $10 \mathrm{E}$ & 0 & 0 & 0 & 0 & 0 & 0 & 0 & 0 & 0 & 0 & 0 & 0 & 0 & 0 & 0 & 0 & 0 & 0 & 0 \\
\hline $10 \mathrm{~F}$ & 0 & 0 & 0 & 0 & 0 & 0 & 0 & 0 & 0 & 0 & 0 & 0 & 0 & 0 & 0 & 0 & 0 & 0 & 0 \\
\hline $10 \mathrm{~N}$ & 0 & 0 & 0 & 0 & 0 & 0 & 0 & 0 & 0 & 0 & 0 & 0 & 0 & 39 & 0 & 0 & 0 & 0 & 0 \\
\hline $10 \mathrm{~s}$ & 0 & 0 & 0 & 0 & 0 & 0 & 0 & 0 & 0 & 0 & 0 & 0 & 0 & 1d & 0 & 0 & 0 & 0 & 0 \\
\hline 10ss & 0 & 의 & 0 & 의 & 0 & 0 & 0 & 0 & 0 & 0 & 0 & 0 & 0 & 1 & 0 & 0 & 0 & 0 & 0 \\
\hline 11 & & & 0 & 0 & 0 & 0 & 0 & 0 & 0 & 0 & 0 & 0 & 0 & 0 & 0 & 0 & 4 & 0 & 0 \\
\hline $11 \mathrm{~N}$ & 35 & 34 & 0 & 0 & of & 0 & 0 & 0 & 0 & 0 & 0 & 0 & 0 & 194 & 1 & 0 & 0 & 0 & 0 \\
\hline $11 R$ & 0 & 60 & 41 & 0 & 29 & 7 & 39 & 0 & 0 & 0 & of & 0 & of & 0 & 0 & 0 & 0 & 0 & of \\
\hline $11 \mathrm{~S}$ & 0 & 0 & 0 & 0 & 0 & 0 & 0 & 0 & 0 & 0 & 0 & 0 & 0 & 20 & 0 & 0 & 0 & 0 & 0 \\
\hline $12 \mathrm{~A}$ & 0 & 0 & & 0 & 0 & 0 & 0 & 0 & 0 & 0 & 0 & 0 & 0 & 0 & 0 & 0 & 0 & 0 & 0 \\
\hline $12 B$ & 0 & 0 & & 0 & 0 & 0 & 0 & 0 & 0 & 0 & of & 0 & 0 & 0 & 0 & 0 & 0 & 0 & 0 \\
\hline $13 \mathrm{C}$ & 56 & 0 & 1 & 0 & 0 & & 0 & 0 & 0 & 0 & 0 & 0 & 4 & 1 & 0 & 0 & 0 & 0 & 0 \\
\hline 13D & 61 & 34 & 41 & 24 & 0 & 2 & 60 & 26 & 29 & 52 & 68) & 38 & 63 & 17 & 11 & 13 & 1 & 21 & 56 \\
\hline 13E & 0 & 3 & 0 & 0 & 1 & 0 & 0 & 0 & 0 & 1 & 0 & 0 & 0 & 15 & 0 & 0 & 0 & 0 & 0 \\
\hline $13 \mathrm{~F}$ & 0 & 2 & 0 & 0 & 67 & 63 & 39 & 0 & 0 & 0 & 0 & 0 & 0 & 0 & 0 & 0 & 0 & 0 & 0 \\
\hline $13 G$ & 62 & 0 & 0 & 0 & 3 & 41 & 0 & 0 & 0 & 247 & 205 & 80 & 60 & 28 & 16 & 19 & 17 & 7 & 18 \\
\hline $13 \mathrm{H}$ & 80 & 73 & 82 & 88 & 53 & 30 & 27 & 64 & 92 & 140 & $10 \mathrm{~d}$ & 98 & 97 & 17 & 8 & 21 & 17 & 23 & 25 \\
\hline 131 & 0 & 3 & 0 & 0 & 0 & 0 & 11. & 122 & 104 & 203 & 139 & 103 & 159 & 41 & 10 & 0 & 0 & 0 & 0 \\
\hline $13 \mathrm{~J}$ & 40 & 0 & 2 & 0 & 2 & 0 & 80 & 79 & 0 & 0 & 91 & 0 & 64 & 25 & 15 & 0 & 22 & 14 & 32 \\
\hline $13 K$ & 45 & 61 & 64 & 20 & 24 & 17 & 24 & 24 & 45 & 74 & 0 & 74 & 0 & 0 & 0 & 0 & 0 & 0 & 0 \\
\hline $13 \mathrm{~L}$ & 0 & 0 & 0 & 0 & 3 & 0 & 0 & 0 & 0 & 0 & 0 & 0 & 0 & 0 & 0 & 0 & 0 & 0 & 0 \\
\hline $13 \mathrm{M}$ & 0 & 0 & 0 & 0 & 0 & 5 & 0 & 0 & 0 & 0 & 0 & 0 & 0 & 281 & 0 & 27 & of & 0 & 0 \\
\hline $13 \mathrm{~N}$ & & & 0 & 0 & 0 & 0 & 0 & 0 & 0 & 0 & 0 & 0 & 0 & 0 & 0 & 27 & 0 & 0 & 0 \\
\hline $13 P$ & 0 & 0 & 0 & 0 & 0 & 2 & 1 & 0 & 0 & 0 & 0 & 0 & 0 & 0 & 0 & 0 & 0 & 0 & 0 \\
\hline 14 & & 0 & 0 & 0 & & 0 & 0 & 0 & 0 & 0 & 0 & 0 & 0 & 0 & 0 & 0 & 0 & 0 & 0 \\
\hline 14 Pond & 0 & 0 & 0 & 7 & 0 & 0 & 0 & 0 & 0 & 0 & 0 & 0 & 0 & 0 & 0 & 0 & 0 & 0 & 0 \\
\hline $14 \mathrm{~A}$ & 14 & 0 & 0 & 0 & 0 & 0 & 0 & 0 & 0 & 0 & 0 & 0 & 0 & 0 & 0 & 0 & 0 & 0 & 0 \\
\hline $14 \mathrm{~B}$ & 16 & 0 & 0 & 0 & 0 & 0 & 0 & 0 & 0 & 0 & 0 & 0 & 0 & 0 & 0 & 0 & 0 & 0 & 0 \\
\hline $14 \mathrm{C}$ & 47 & 21 & 2 & 20 & 12 & 21 & 20 & 16 & 0 & 0 & 0 & 0 & 0 & 0 & 0 & 0 & 0 & 0 & 0 \\
\hline 14D & 0 & 0 & 43 & 35 & 32 & 15 & 18 & 35 & 0 & 1 & 122 & 0 & 0 & 0 & 31 & 1 & 0 & 0 & 0 \\
\hline 14E & 0 & 0 & 0 & 84 & 98 & 37) & 48 & 42 & 0 & 0 & 138 & 257 & 143 & 0 & 111 & 108 & 78 & 67 & 86 \\
\hline $14 \mathrm{~F}$ & 59 & 75 & 0 & 65 & 73 & 24 & 20 & 50 & 0 & 0 & 162 & 208 & 169 & 0 & 37 & 83 & 35 & 28 & 35 \\
\hline $14 \mathrm{G}$ & 0 & 0 & 0 & 0 & 0 & 1 & 13 & 37 & 0 & 2 & 0 & 0 & 1 & 0 & 0 & 0 & 0 & 0 & 0 \\
\hline $14 \mathrm{H}$ & 0 & 0 & 61 & 0 & 0 & 24 & 28 & 44 & 74 & 74 & 0 & 0 & 0 & 0 & 13 & 0 & 0 & 0 & 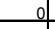 \\
\hline 141 & 44 & 0 & 61 & 40 & 21 & 17 & 32 & 61 & 32 & 77 & 98 & 159 & 110 & 0 & 80 & 98 & 63 & 48 & 54 \\
\hline $14 \mathrm{~J}$ & 0 & 16 & 39 & 0 & 0 & 0 & 0 & 0 & 0 & 8 & 0 & 0 & 0 & 0 & 44 & 69 & 51 & 0 & 47 \\
\hline $14 \mathrm{~K}$ & 0 & 0 & 0 & 0 & 1 & 0 & 0 & 0 & 0 & 0 & 0 & 0 & 0 & 0 & 2 & 0 & 0 & 0 & 0 \\
\hline $14 \mathrm{~L}$ & 17 & 74 & 69 & 57 & 44 & 12 & 5 & 0 & 0 & 0 & 0 & 0 & 0 & 0 & 17 & 0 & 25 & 0 & 0 \\
\hline $14 \mathrm{M}$ & 0 & 48 & 11. & 85 & 67 & 51 & 75 & 44 & 82 & $15 \mathrm{~S}$ & 0 & 0 & 0 & 0 & 34 & 0 & 82 & 79 & 48 \\
\hline $14 \mathrm{~N}$ & 0 & 92 & 40 & 90 & 91 & 0 & 2 & 157 & 0 & 3 & 0 & 128 & 22 & 0 & 30 & 50 & 0 & 0 & 0 \\
\hline 140 & 0 & 46 & 11. & 73 & 90 & 62 & 53 & 83 & 91 & $13 \mathrm{~g}$ & 0 & 114 & 53 & 6 & 38 & 411 & 44 & 22 & 0 \\
\hline $14 \mathrm{P}$ & 0 & 0 & 7 & 0 & 0 & 0 & 1 & 0 & 0 & 0 & 0 & 0 & 0 & 8 & 0 & 0 & 0 & 0 & 0 \\
\hline 15 & 0 & 66 & 0 & 9 & 0 & 0 & 0 & 0 & 0 & 0 & 0 & 0 & 0 & 0 & 0 & 0 & of & 0 & 0 \\
\hline $15 \mathrm{~A}$ & 0 & 0 & 1 & 0 & 0 & 0 & 0 & 0 & 0 & 0 & 0 & 0 & 0 & 0 & 0 & 0 & 0 & 0 & 0 \\
\hline $15 \mathrm{mid}$ & 0 & 0 & 0 & 0 & 0 & 0 & 0 & 0 & 0 & 0 & 0 & 0 & 0 & 0 & 0 & 0 & 2 & 0 & 0 \\
\hline $15 \mathrm{~N}$ & 0 & 0 & 0 & 0 & 0 & 0 & 0 & 0 & 0 & 0 & 0 & 0 & 0 & 87 & 15 & 25 & 0 & 0 & 0 \\
\hline $15 \mathrm{~N}$ Leve & 0 & 0 & 0 & of & 0 & 0 & 0 & 0 & 0 & 0 & 0 & 0 & 0 & 4 & 0 & 0 & 0 & 0 & 0 \\
\hline $15 \mathrm{~N} \mathrm{mid}$ & 0 & 0 & 0 & & 0 & 0 & 0 & 0 & 0 & 0 & 0 & 0 & 0 & 0 & 0 & 18 & 0 & 0 & 0 \\
\hline $15 \mathrm{NN}$ & 0 & 0 & 0 & 0 & 0 & 0 & 0 & 0 & 0 & 0 & of & 0 & 0 & 82 & 0 & 0 & of & 0 & 0 \\
\hline 15NN Levee & 0 & 0 & 0 & & 0 & 0 & 0 & 0 & 0 & 0 & 0 & 0 & 0 & 4 & 0 & 0 & 0 & 0 & 0 \\
\hline $15 \mathrm{~S}$ & 0 & 0 & 0 & & & & 0 & 0 & 0 & 0 & 0 & 0 & 0 & 71 & 7 & 14 & & 0 & 0 \\
\hline $15 \mathrm{~W}$ & 0 & 0 & 0 & 0 & 0 & 0 & 0 & 0 & 0 & 0 & 0 & 0 & 0 & 60 & 12 & 0 & 0 & 0 & 0 \\
\hline $17 \mathrm{~A}$ & 0 & 0 & 0 & 0 & 1 & 0 & 0 & 0 & 0 & 0 & 0 & 0 & 0 & 0 & 0 & 0 & 0 & 0 & 0 \\
\hline 17D & 0 & 0 & 0 & 11 & 5 & 18 & 0 & 0 & 0 & 0 & 0 & 0 & 0 & 0 & 0 & 0 & 0 & 0 & 0 \\
\hline $17 E$ & 0 & 0 & 0 & 0 & 10 & 5 & 0 & 0 & 0 & 0 & 0 & 0 & 0 & 0 & 0 & 0 & 0 & 0 & 0 \\
\hline 2 & 0 & 0 & 0 & 1 & 0 & 0 & 0 & 0 & 0 & 0 & 0 & 0 & 0 & 0 & 0 & 0 & 0 & 0 & 0 \\
\hline $22 \mathrm{~A}$ & & 0 & 0 & & & 4 & 0 & 0 & 0 & 0 & 0 & 0 & 0 & 0 & 0 & 0 & 0 & 0 & 0 \\
\hline $23 \mathrm{~A}$ & 0 & 18 & 0 & 0 & 0 & 0 & 0 & 0 & 0 & 0 & 0 & 0 & 0 & 0 & 0 & 0 & 0 & 0 & 0 \\
\hline $23 B$ & 0 & 15 & 0 & & 0 & 0 & 0 & 0 & 0 & 0 & 0 & 0 & 0 & 0 & 0 & 0 & & 0 & 0 \\
\hline $3 \mathrm{E}$ & & 0 & 10 & & 0 & 0 & 0 & 0 & 0 & 0 & 0 & 0 & d & 0 & 0 & 0 & 0 & 0 & 0 \\
\hline 4 & 0 & 0 & 0 & 0 & 5 & 4 & 0 & 0 & 0 & 0 & 0 & 0 & 0 & 0 & 0 & 0 & 0 & 0 & 0 \\
\hline 5 & 0 & 0 & 0 & 6 & 6 & & 0 & 0 & 0 & 0 & 0 & 0 & , & 0 & 0 & 0 & 0 & 0 & 0 \\
\hline 6 & 0 & 0 & 0 & 1 & 0 & 0 & 0 & 0 & 0 & 0 & 0 & 0 & 0 & 0 & 0 & 0 & 0 & 0 & of \\
\hline 8 & 0 & 0 & 0 & & 7 & & 0 & 0 & 0 & 0 & 0 & 0 & 0 & 0 & 0 & 0 & 0 & 0 & 0 \\
\hline & 0 & 0 & 0 & 1 & 0 & 0 & 0 & 0 & 0 & 0 & 0 & 0 & 0 & 0 & 0 & 0 & 0 & 0 & 0 \\
\hline Crescent Unit & & & 0 & & 0 & 0 & 0 & 0 & 0 & 0 & 0 & 0 & 0 & 0 & 0 & 0 & 0 & 0 & 0 \\
\hline Dike & & 0 & 0 & & 0 & 0 & & 0 & & 0 & 0 & 0 & & & & 0 & & 0 & 0 \\
\hline DUA & 0 & 0 & 0 & 0 & 0 & 2 & 0 & 0 & 0 & 0 & 0 & 0 & 0 & 0 & 0 & 0 & 0 & 0 & 0 \\
\hline GRA & 0 & 0 & 0 & 0 & 0 & 0 & 0 & 0 & 0 & 0 & 0 & 0 & 0 & 0 & 0 & 0 & 0 & 0 & 0 \\
\hline GRB & 0 & 0 & 0 & 0 & 0 & 0 & 0 & 0 & 0 & 0 & 0 & 0 & 0 & 0 & 0 & 0 & 0 & 0 & 0 \\
\hline Incidental & 0 & 6 & 0 & 1 & 0 & 11 & 13 & 6 & 0 & 0 & 3 & 0 & 0 & 0 & 0 & 0 & 0 & 0 & 0 \\
\hline ISD & 0 & 0 & 0 & 0 & 0 & 5 & 0 & 0 & 0 & 0 & 0 & 0 & 0 & 0 & 0 & 0 & 0 & 0 & 0 \\
\hline $\mathrm{JOI}$ & & & & & & & & & & & 0 & & & & 0 & 0 & & & \\
\hline Total & 587 & 758 & 812 & 724 & 746 & 483 & 716 & 890 & 549 & 1176 & 1129 & 1259 & 945 & 764 & 533 & 614 & 452 & 309 & 401 \\
\hline
\end{tabular}

Figure 9. Summary of all waterfowl and associated species nest data collected by field at Grizzly Island Wildlife Area, California, 1985-2014. 
Appendix A. Archival Metadata, Including File Names, Location of Data, Site Names, and Years

Appendix A is a Microsoft巴 Excel file that can be downloaded at http://pubs.usgs.gov/of/2015/1131/. 


\section{Appendix B. Summary of All Duck Nest Data Collected by Study Region, Field, and Year, California, 1985-2014}

" 0 " either means the field was searched but no nests were found, or that the field was not searched in that specific year.

\begin{tabular}{|c|c|c|c|c|}
\hline Site & $\begin{array}{l}\text { Year } \\
1994\end{array}$ & 1995 & 1996 & 1997 \\
\hline Goose Pond A & 5 & 2 & 7 & $\overline{0}$ \\
\hline Goose Pond B & 9 & 8 & 5 & 1 \\
\hline Hutchinson Creek & 6 & 0 & 0 & 4 \\
\hline Hutchinson Creek South & 0 & 0 & 6 & 0 \\
\hline Incidental & 0 & 1 & 0 & 0 \\
\hline Lower Miller & 3 & 0 & 2 & 3 \\
\hline Mad Dog North & 0 & 1 & 0 & 0 \\
\hline MD & 0 & 0 & 1 & 0 \\
\hline Parks Lake & 0 & 0 & 0 & 22 \\
\hline Pheasant Farm & 0 & 1 & 1 & o \\
\hline Upper Black Welder & 8 & 0 & 5 & \\
\hline
\end{tabular}

Butte Valley Wildlife Area

Year

\begin{tabular}{rr} 
Site & 1988 \\
\hline $4 \mathrm{~A}$ & 45
\end{tabular}

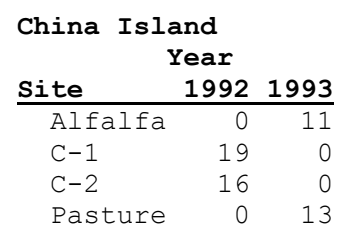

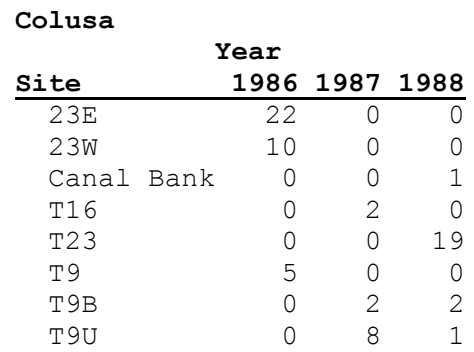

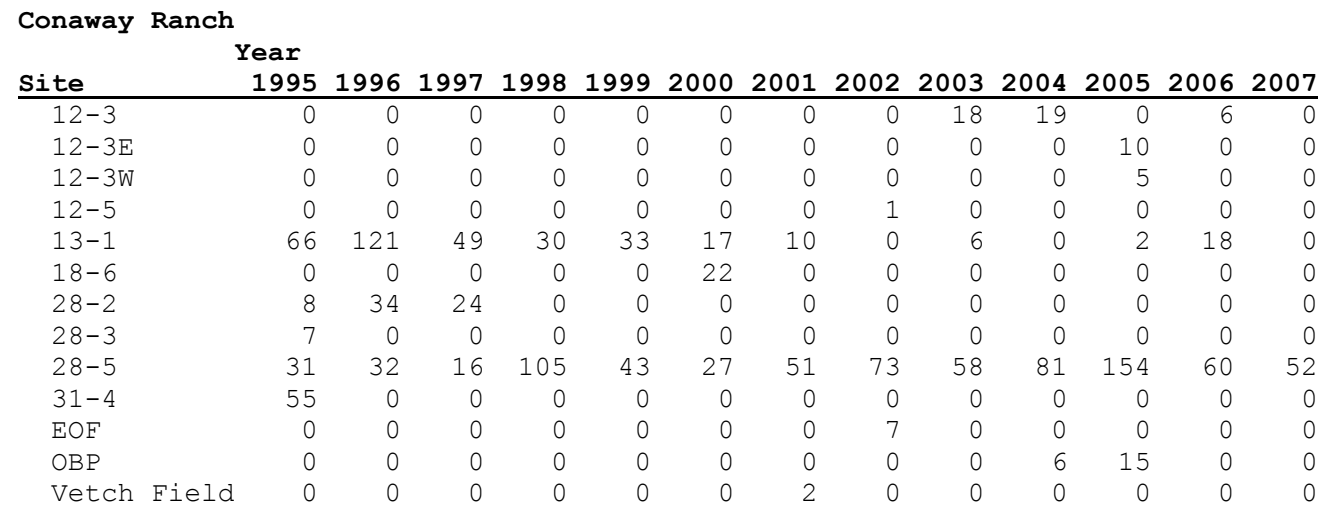




\begin{tabular}{lrrrrr} 
CREP - Colusa County \\
Year \\
Site & $\mathbf{2 0 0 2}$ & $\mathbf{2 0 0 3}$ & $\mathbf{2 0 0 4}$ & $\mathbf{2 0 0 5}$ & $\mathbf{2 0 0 6}$ \\
\hline 4 mile Road & 0 & 0 & 15 & 0 & 0 \\
Buckhorn & 0 & 2 & 0 & 7 & 2 \\
Colusa NWR & 0 & 11 & 20 & 25 & 0 \\
Finch East & 0 & 0 & 0 & 27 & 0 \\
Finch West & 0 & 0 & 0 & 20 & 19 \\
Kise & 2 & 8 & 0 & 5 & 6 \\
La Grange & 0 & 0 & 0 & 16 & 0 \\
Rogers Wheat & 0 & 0 & 0 & 22 & 0 \\
Ryan & 0 & 0 & 0 & 0 & 56 \\
Traynham Ranch & 0 & 0 & 0 & 0 & 3 \\
White Road & 0 & 5 & 4 & 10 & 0 \\
White Road East & 4 & 0 & 0 & 0 & 0 \\
White Road West & 6 & 0 & 0 & 0 & 0
\end{tabular}

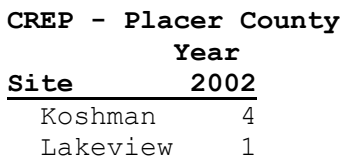

\begin{tabular}{lrrrrr} 
CREP - Yolo County & & & & \\
Year \\
Site & $\mathbf{2 0 0 2}$ & $\mathbf{2 0 0 3}$ & $\mathbf{2 0 0 4}$ & $\mathbf{2 0 0 5}$ & $\mathbf{2 0 0 6}$ \\
\hline Dyer & 0 & 0 & 0 & 0 & 8 \\
Dyer Wheat & 0 & 3 & 0 & 0 & 0 \\
Keeley & 0 & 0 & 55 & 0 & 0 \\
Lillard & 0 & 0 & 20 & 18 & 20 \\
Long & 0 & 5 & 4 & 5 & 0 \\
Long Wheat & 0 & 0 & 0 & 0 & 20 \\
Newman & 4 & 22 & 0 & 0 & 6 \\
Newman N & 0 & 0 & 10 & 14 & 0 \\
Newman S & 0 & 0 & 21 & 1 & 0 \\
Olevario & 0 & 1 & 0 & 0 & 6 \\
Ottenwalter & 0 & 0 & 18 & 0 & 0 \\
Van Tram & 0 & 0 & 0 & 0 & 4 \\
Wallace & 14 & 0 & 0 & 0 & 0
\end{tabular}
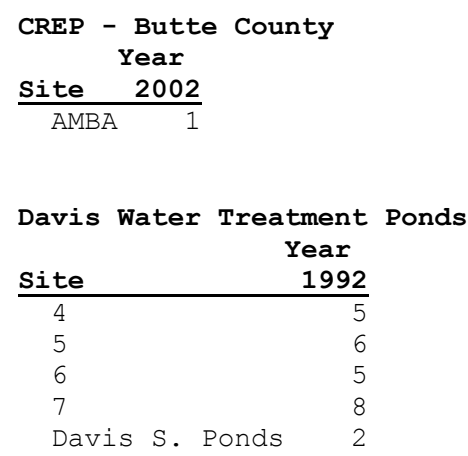

\begin{tabular}{crrrr}
$\begin{array}{c}\text { Delevan National } \\
\text { Year } \\
\text { Site }\end{array}$ & $\mathbf{1 9 8 6}$ & $\mathbf{1 9 8 7}$ & $\mathbf{1 9 8 8}$ & $\mathbf{2 0 0 8}$ \\
\hline $3 / 4$ & 53 & 0 & 9 & 0 \\
35 & 0 & 0 & 13 & 0 \\
37 & 34 & 43 & 19 & 0 \\
41 & 0 & 36 & 56 & 1 \\
D43 & 0 & 0 & 6 & 0 \\
Incidental & 0 & 0 & 1 & 0 \\
T9 & 0 & 0 & 5 & 0
\end{tabular}




\begin{tabular}{|c|c|c|c|c|c|}
\hline \multicolumn{4}{|c|}{ Denverton } & & \\
\hline \multicolumn{6}{|c|}{$\begin{array}{c}\text { Year } \\
\text { Site } 2013\end{array}$} \\
\hline T3 & \multicolumn{5}{|l|}{$\frac{5}{1}$} \\
\hline $\mathrm{T} 4$ & \multicolumn{5}{|l|}{1} \\
\hline \multicolumn{6}{|l|}{ Fendt } \\
\hline \multicolumn{6}{|c|}{ Year } \\
\hline Site & 2002 & 2003 & 2004 & 2005 & 2006 \\
\hline 1 & 0 & 80 & 29 & 0 & $\overline{0}$ \\
\hline East & 0 & 0 & 0 & 11 & 0 \\
\hline Fallow & 36 & 0 & 0 & 0 & 0 \\
\hline Rice & 7 & 0 & 0 & 0 & 0 \\
\hline West & 0 & 0 & 0 & 65 & 76 \\
\hline Wheat & 0 & 0 & 0 & 0 & 46 \\
\hline
\end{tabular}

Garibaldi

Year

Site 1988

$\begin{array}{ll}1 & 2 \\ 2 & 3\end{array}$

\begin{tabular}{lrrrrrrrr} 
Gray Lodge Wildlife & Area \\
Year & & & & & & & \\
Site & $\mathbf{1 9 8 6}$ & $\mathbf{1 9 8 7}$ & $\mathbf{1 9 8 8}$ & $\mathbf{1 9 9 7}$ & $\mathbf{1 9 9 8}$ & $\mathbf{1 9 9 9}$ & $\mathbf{2 0 0 0}$ & $\mathbf{2 0 0 1}$ \\
\hline 13 & 0 & 0 & 5 & 0 & 0 & 0 & 0 & 0 \\
19 & 6 & 7 & 3 & 0 & 0 & 0 & 0 & 0 \\
22 & 2 & 0 & 0 & 0 & 0 & 0 & 0 & 0 \\
28 & 0 & 0 & 1 & 0 & 0 & 0 & 0 & 0 \\
31 & 1 & 0 & 0 & 0 & 0 & 0 & 0 & 0 \\
39 & 1 & 0 & 0 & 0 & 0 & 0 & 0 & 0 \\
42 & 0 & 14 & 12 & 0 & 0 & 0 & 0 & 0 \\
45 & 1 & 0 & 0 & 0 & 0 & 0 & 0 & 0 \\
51 & 0 & 15 & 20 & 0 & 0 & 0 & 4 & 0 \\
52 & 0 & 0 & 3 & 5 & 0 & 0 & 0 & 1 \\
$52 \mathrm{~A}$ & 0 & 6 & 7 & 0 & 0 & 0 & 0 & 0 \\
$52 \mathrm{~B}$ & 0 & 2 & 0 & 0 & 0 & 0 & 0 & 0 \\
57 & 1 & 9 & 0 & 0 & 0 & 0 & 0 & 0 \\
$57 \mathrm{~N}$ & 4 & 0 & 0 & 0 & 0 & 0 & 0 & 0 \\
$57 \mathrm{~S}$ & 3 & 0 & 0 & 0 & 0 & 0 & 0 & 0 \\
58 & 15 & 26 & 4 & 0 & 0 & 0 & 0 & 0 \\
$62 \mathrm{~W}$ & 6 & 0 & 0 & 0 & 0 & 0 & 0 & 0 \\
63 & 0 & 7 & 2 & 0 & 0 & 0 & 0 & 0 \\
$63 \mathrm{~N}$ & 9 & 0 & 0 & 0 & 0 & 0 & 0 & 0 \\
$63 \mathrm{~S}$ & 10 & 0 & 0 & 0 & 0 & 0 & 0 & 0 \\
66 & 6 & 0 & 0 & 0 & 0 & 0 & 0 & 0 \\
67 & 0 & 0 & 1 & 0 & 0 & 0 & 0 & 0 \\
74 & 0 & 0 & 0 & 0 & 10 & 10 & 4 & 0 \\
79 & 0 & 0 & 0 & 0 & 0 & 4 & 0 & 0 \\
81 & 7 & 0 & 0 & 0 & 0 & 0 & 0 & 0 \\
82 & 0 & 35 & 47 & 10 & 28 & 15 & 9 & 8 \\
84 & 7 & 3 & 0 & 0 & 1 & 0 & 0 & 0 \\
85 & 0 & 4 & 0 & 0 & 0 & 0 & 0 & 0 \\
90 & 12 & 9 & 0 & 0 & 0 & 0 & 0 & 0 \\
91 & 1 & 0 & 0 & 0 & 0 & 0 & 0 & 0 \\
92 & 0 & 0 & 0 & 0 & 0 & 0 & 0 & 1 \\
BP-52 & 0 & 0 & 0 & 7 & 0 & 0 & 0 & 0 \\
BPA & 0 & 0 & 0 & 0 & 2 & 0 & 0 & 0 \\
BPB & 0 & 0 & 0 & 0 & 2 & 0 & 0 & 0 \\
Incidental & 7 & 24 & 0 & 0 & 0 & 0 & 0 & 0 \\
Justeson & 0 & 6 & 0 & 0 & 0 & 0 & 0 & 0
\end{tabular}


Grizzly Island Wildlife Area

\section{Year}

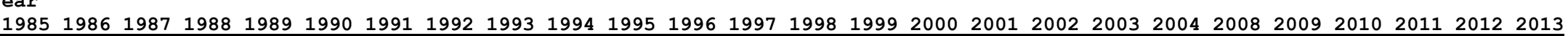

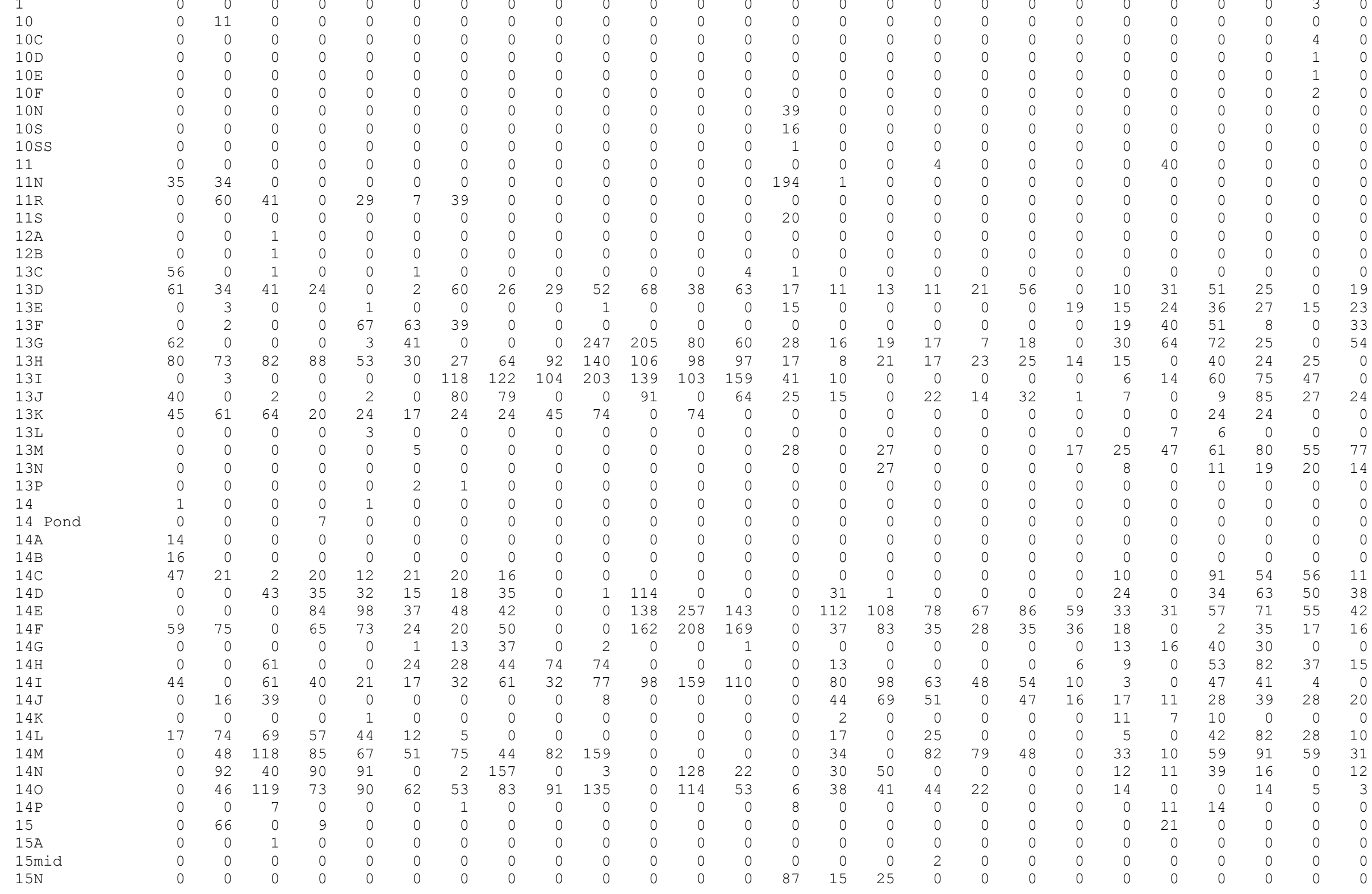


Grizzly Island Wildlife Area (continued)

\begin{tabular}{|c|c|c|c|c|c|c|c|c|c|c|c|c|c|c|c|c|c|c|c|c|c|c|c|c|c|c|}
\hline te & $\begin{array}{l}\text { lear } \\
1985\end{array}$ & 1986 & 1987 & 1988 & 1989 & 1990 & 1991 & 1992 & 1993 & 1994 & 1995 & 1996 & 1997 & 1998 & 1999 & 2000 & 2001 & 2002 & 2003 & 2004 & 2008 & 2009 & 2010 & 2011 & 2012 & 2013 \\
\hline $15 \mathrm{~N}$ Levee & 0 & 0 & 0 & 0 & 0 & 0 & 0 & 0 & 0 & 0 & 0 & 0 & 0 & 4 & 0 & 0 & 0 & 0 & 0 & 0 & 0 & 0 & $\frac{0}{0}$ & 0 & 0 & 0 \\
\hline $15 \mathrm{~N}$ mid & 0 & 0 & 0 & 0 & 0 & 0 & 0 & 0 & 0 & 0 & 0 & 0 & 0 & 0 & 0 & 18 & 0 & 0 & 0 & 0 & 0 & 0 & 0 & 0 & 0 & 0 \\
\hline $15 \mathrm{NN}$ & 0 & 0 & 0 & 0 & 0 & 0 & 0 & 0 & 0 & 0 & 0 & 0 & 0 & 82 & 0 & 0 & 0 & 0 & 0 & 0 & 0 & 0 & 0 & 0 & 0 & 0 \\
\hline 15NN Levee & 0 & 0 & 0 & 0 & 0 & 0 & 0 & 0 & 0 & 0 & 0 & 0 & 0 & 4 & 0 & 0 & 0 & 0 & 0 & 0 & 0 & 0 & 0 & 0 & 0 & 0 \\
\hline $15 \mathrm{~S}$ & 0 & 0 & 0 & 0 & 0 & 0 & 0 & 0 & 0 & 0 & 0 & 0 & 0 & 71 & 7 & 14 & 1 & 0 & 0 & 0 & 0 & 0 & 0 & 0 & 0 & 0 \\
\hline $15 \mathrm{~W}$ & 0 & 0 & 0 & 0 & 0 & 0 & 0 & 0 & 0 & 0 & 0 & 0 & 0 & 60 & 12 & 0 & 0 & 0 & 0 & 0 & 0 & 0 & 0 & 0 & 0 & 0 \\
\hline $17 \mathrm{~A}$ & 0 & 0 & 0 & 0 & 1 & 0 & 0 & 0 & 0 & 0 & 0 & 0 & 0 & 0 & 0 & 0 & 0 & 0 & 0 & 0 & 0 & 0 & 0 & 0 & 0 & 0 \\
\hline $17 \mathrm{D}$ & 0 & 0 & 0 & 11 & 5 & 18 & 0 & 0 & 0 & 0 & 0 & 0 & 0 & 0 & 0 & 0 & 0 & 0 & 0 & 0 & 0 & 0 & 0 & 0 & 0 & 0 \\
\hline $17 \mathrm{E}$ & 0 & 0 & 0 & 0 & 10 & 5 & 0 & 0 & 0 & 0 & 0 & 0 & 0 & 0 & 0 & 0 & 0 & 0 & 0 & 0 & 0 & 0 & 0 & 0 & 0 & 0 \\
\hline 2 & 0 & 0 & 0 & 1 & 0 & 0 & 0 & 0 & 0 & 0 & 0 & 0 & 0 & 0 & 0 & 0 & 0 & 0 & 0 & 0 & 0 & 0 & 0 & 0 & 3 & 0 \\
\hline $22 \mathrm{~A}$ & 1 & 0 & 0 & 0 & 0 & 4 & 0 & 0 & 0 & 0 & 0 & 0 & 0 & 0 & 0 & 0 & 0 & 0 & 0 & 0 & 0 & 0 & 0 & 0 & 0 & 0 \\
\hline $23 \mathrm{~A}$ & 0 & 18 & 0 & 0 & 0 & 0 & 0 & 0 & 0 & 0 & 0 & 0 & 0 & 0 & 0 & 0 & 0 & 0 & 0 & 0 & 0 & 0 & 0 & 0 & 0 & 0 \\
\hline $23 \mathrm{~B}$ & 0 & 15 & 0 & 0 & 0 & 0 & 0 & 0 & 0 & 0 & 0 & 0 & 0 & 0 & 0 & 0 & 0 & 0 & 0 & 0 & 0 & 0 & 0 & 0 & 0 & 0 \\
\hline $3 E$ & 0 & 0 & 16 & 0 & 0 & 0 & 0 & 0 & 0 & 0 & 0 & 0 & 0 & 0 & 0 & 0 & 0 & 0 & 0 & 0 & 0 & 0 & 0 & 0 & 0 & \\
\hline 4 & 0 & 0 & 0 & 0 & 5 & 4 & 0 & 0 & 0 & 0 & 0 & 0 & 0 & 0 & 0 & 0 & 0 & 0 & 0 & 0 & 0 & 0 & 0 & 0 & 1 & 0 \\
\hline 5 & 0 & 0 & 0 & 6 & 6 & 1 & 0 & 0 & 0 & 0 & 0 & 0 & 0 & 0 & 0 & 0 & 0 & 0 & 0 & 0 & 0 & 0 & 0 & 0 & 1 & 0 \\
\hline 6 & 0 & 0 & 0 & 1 & 0 & 0 & 0 & 0 & 0 & 0 & 0 & 0 & 0 & 0 & 0 & 0 & 0 & 0 & 0 & 0 & 0 & 0 & 0 & 0 & 0 & 0 \\
\hline 8 & 0 & 0 & 0 & 6 & 7 & 1 & 0 & 0 & 0 & 0 & 0 & 0 & 0 & 0 & 0 & 0 & 0 & 0 & 0 & 0 & 0 & 0 & 0 & 0 & 0 & 0 \\
\hline $8 B$ & 0 & 0 & 0 & 1 & 0 & 0 & 0 & 0 & 0 & 0 & 0 & 0 & 0 & 0 & 0 & 0 & 0 & 0 & 0 & 0 & 0 & 0 & 0 & 0 & 0 & 0 \\
\hline Crescent Unit & 8 & 0 & 0 & 0 & 0 & 0 & 0 & 0 & 0 & 0 & 0 & 0 & 0 & 0 & 0 & 0 & 0 & 0 & 0 & 0 & 0 & 0 & 0 & 0 & 0 & \\
\hline Dike & 1 & 0 & 0 & 0 & 0 & 0 & 0 & 0 & 0 & 0 & 0 & 0 & 0 & 0 & 0 & 0 & 0 & 0 & 0 & 0 & 0 & 0 & 0 & 0 & 0 & \\
\hline DUA & 0 & 0 & 0 & 0 & 0 & 2 & 0 & 0 & 0 & 0 & 0 & 0 & 0 & 0 & 0 & 0 & 0 & 0 & 0 & 0 & 0 & 0 & 0 & 0 & 0 & 0 \\
\hline GRA & 0 & 0 & 0 & 0 & 0 & 0 & 0 & 0 & 0 & 0 & 0 & 0 & 0 & 0 & 0 & 0 & 0 & 0 & 0 & 0 & 0 & 0 & 0 & 0 & 0 & \\
\hline GRB & 0 & 0 & 0 & 0 & 0 & 0 & 0 & 0 & 0 & 0 & 0 & 0 & 0 & 0 & 0 & 0 & 0 & 0 & 0 & 0 & 0 & 0 & 0 & 0 & 0 & \\
\hline Incidental & 0 & 6 & 0 & 1 & 0 & 11 & 13 & 6 & 0 & 0 & 0 & 0 & 0 & 0 & 0 & 0 & 0 & 0 & 0 & 0 & 0 & 0 & 0 & 0 & 0 & \\
\hline ISD & 0 & 0 & 0 & 0 & 0 & 5 & 0 & 0 & 0 & 0 & 0 & 0 & 0 & 0 & 0 & 0 & 0 & 0 & 0 & 0 & 0 & 0 & 0 & 0 & 0 & \\
\hline JOI & 0 & 0 & 3 & 0 & 0 & 0 & 0 & 0 & 0 & 0 & 0 & 0 & 0 & 0 & 0 & 0 & 0 & 0 & 0 & 0 & 0 & 0 & 0 & 0 & 0 & \\
\hline
\end{tabular}




\begin{tabular}{|c|c|c|c|}
\hline $\begin{array}{l}\text { Honey Lake / } \\
\text { Site }\end{array}$ & $\begin{array}{c}\text { Ash } \\
\text { Year } \\
1987\end{array}$ & $\begin{array}{l}\text { Creek } \\
1988 \\
\end{array}$ & $\begin{array}{l}\text { WAs } \\
1989 \\
\end{array}$ \\
\hline 1 & 19 & 5 & 107 \\
\hline $11 \mathrm{R}$ & 0 & 3 & 0 \\
\hline 13 & 0 & 4 & 0 \\
\hline $14-2$ & 0 & 3 & 0 \\
\hline 15 & 0 & 9 & 0 \\
\hline $15-16$ & 0 & 0 & 3 \\
\hline 16 & 0 & 2 & 0 \\
\hline 18 & 0 & 10 & 0 \\
\hline 2 & 7 & 0 & 7 \\
\hline $2 \mathrm{E}$ & 0 & 4 & 0 \\
\hline 3 & 8 & 4 & 1 \\
\hline 4 & 33 & 26 & 29 \\
\hline 5 & 5 & 0 & 2 \\
\hline $5 A$ & 1 & 7 & 2 \\
\hline $5 A / 5 B$ & 0 & 0 & 3 \\
\hline $5 \mathrm{~A} 2$ & 14 & 0 & 0 \\
\hline $5 \mathrm{C}$ & 0 & 7 & 0 \\
\hline $5 C / 5 B$ & 0 & 1 & 1 \\
\hline 6 & 4 & 7 & 0 \\
\hline $6 \mathrm{~A} 2$ & 0 & 0 & 17 \\
\hline $6 \mathrm{C}$ & 4 & 1 & 0 \\
\hline $6 \mathrm{C} 1$ & 0 & 0 & 4 \\
\hline $6 \mathrm{C} 2$ & 0 & 0 & 38 \\
\hline $6 F$ & 0 & 3 & 0 \\
\hline $6 \mathrm{G}$ & 0 & 5 & 0 \\
\hline $6 \mathrm{~L}$ & 0 & 3 & 0 \\
\hline 7 & 0 & 35 & 0 \\
\hline 8 & 1 & 0 & 0 \\
\hline A4 & 1 & 0 & 0 \\
\hline AHB & 0 & 0 & 1 \\
\hline D1B & 0 & 0 & 1 \\
\hline $\mathrm{D} 1 \mathrm{C}$ & 0 & 0 & 1 \\
\hline D3A & 41 & 0 & 0 \\
\hline D3B & 41 & 0 & 16 \\
\hline $\mathrm{D} 4 \mathrm{~B}$ & 0 & 1 & 10 \\
\hline D5A & 0 & 0 & 2 \\
\hline $\mathrm{D} 6 \mathrm{~A}$ & 33 & 42 & 0 \\
\hline D6B & 21 & 6 & 0 \\
\hline $\mathrm{D} 6 \mathrm{C}$ & 107 & 27 & 4 \\
\hline $\mathrm{D} 6 \mathrm{E}$ & 0 & 3 & 1 \\
\hline $\mathrm{D} 6 \mathrm{~F}$ & 0 & 0 & 3 \\
\hline $\mathrm{D} 6 \mathrm{G}$ & 0 & 0 & 34 \\
\hline $\mathrm{D} 6 \mathrm{H}$ & 41 & 0 & 11 \\
\hline DGG & 3 & 0 & 0 \\
\hline DL 6 & 0 & 13 & 0 \\
\hline F11 & 0 & 0 & 9 \\
\hline F12 & 0 & 0 & 52 \\
\hline F16 & 0 & 0 & 3 \\
\hline F18 & 0 & 0 & 10 \\
\hline F5A & 1 & 0 & 9 \\
\hline F6B & 7 & 11 & 0 \\
\hline $\mathrm{F} 6 \mathrm{C}$ & 0 & 3 & 0 \\
\hline E7A & 0 & 0 & 17 \\
\hline F7C & 0 & 6 & 0 \\
\hline $\mathrm{F} 8 \mathrm{~A}$ & 6 & 0 & 0 \\
\hline H Res & 0 & 12 & 1 \\
\hline Incidental & 5 & 0 & 1 \\
\hline L6 & 0 & 7 & 0 \\
\hline
\end{tabular}




\begin{tabular}{lrrrrr} 
Kalfsbeek & Year & & & \\
Site & $\mathbf{2 0 0 2}$ & $\mathbf{2 0 0 3}$ & $\mathbf{2 0 0 4}$ & $\mathbf{2 0 0 5}$ & $\mathbf{2 0 0 6}$ \\
\hline N & 5 & 14 & 0 & 0 & 0 \\
N & 0 & 0 & 4 & 13 & 0 \\
None provided & 0 & 0 & 0 & 0 & 15 \\
S & 0 & 0 & 4 & 17 & 0 \\
Wheat & 0 & 0 & 5 & 12 & 27
\end{tabular}

Los Banos Wildlife Area
Year
\begin{tabular}{crrrr} 
Site & $\mathbf{1 9 8 9}$ & $\mathbf{1 9 9 0}$ & $\mathbf{1 9 9 2}$ & $\mathbf{1 9 9 3}$ \\
\hline $26 \mathrm{~A}$ & 0 & 0 & 13 & 0 \\
$26 \mathrm{~B}$ & 0 & 0 & 14 & 0 \\
41 & 0 & 0 & 0 & 12 \\
56 & 92 & 0 & 0 & 0 \\
57 & 0 & 0 & 3 & 0 \\
63 & 0 & 0 & 0 & 1 \\
64 & 0 & 0 & 0 & 25 \\
640D1 & 0 & 0 & 6 & 0 \\
HOL & 0 & 13 & 0 & 0 \\
SW64 & 0 & 0 & 0 & 12
\end{tabular}

\begin{tabular}{|c|c|c|c|}
\hline Mande & $\begin{array}{l}\text { ville } \\
\text { Year }\end{array}$ & Isla & \\
\hline Site & 1988 & 1991 & 2004 \\
\hline 1 & 3 & $\overline{0}$ & 3 \\
\hline 15 & 0 & 0 & 12 \\
\hline 19 & 0 & 0 & 63 \\
\hline $29 \mathrm{M}$ & 0 & 0 & 12 \\
\hline $29 \mathrm{~N}$ & 0 & 0 & 6 \\
\hline $29 \mathrm{~S}$ & 0 & 0 & 4 \\
\hline 3 & 2 & 0 & c \\
\hline $3 E$ & 0 & 0 & 5 \\
\hline $3 \mathrm{~W}$ & 0 & 0 & $\varepsilon$ \\
\hline CD1 & 0 & 3 & c \\
\hline IN1 & 1 & 0 & c \\
\hline
\end{tabular}




\begin{tabular}{|c|c|c|c|c|c|}
\hline Mendota & $\begin{array}{r}\text { Wildlife } \\
\text { Year }\end{array}$ & Area & & & \\
\hline Site & 1987 & 1988 & 1989 & 1990 & 1991 \\
\hline 1 & 0 & 13 & 0 & 0 & $\overline{0}$ \\
\hline 1.2 & 0 & 0 & 22 & 27 & 6 \\
\hline 1.4 & 0 & 0 & 14 & 0 & 0 \\
\hline 11 & 3 & 0 & 0 & 0 & 0 \\
\hline 11.4 & 0 & 0 & 4 & 19 & 9 \\
\hline $11 \mathrm{~A}$ & 5 & 0 & 1 & 0 & 0 \\
\hline 12 & 1 & 0 & 0 & 0 & 0 \\
\hline $12 \mathrm{~A}$ & 1 & 0 & 0 & 0 & 0 \\
\hline 13.1 & 0 & 0 & 5 & 0 & 0 \\
\hline 13.4 & 0 & 0 & 4 & 0 & 0 \\
\hline $13 \mathrm{~A}$ & 1 & 0 & 0 & 0 & 0 \\
\hline $13 D$ & 3 & 0 & 0 & 0 & 0 \\
\hline $13 E$ & 12 & 0 & 0 & 0 & 0 \\
\hline 14.1 & 0 & 0 & 0 & 14 & 2 \\
\hline $14 \mathrm{~A}$ & 1 & 0 & 0 & 0 & 0 \\
\hline 17.7 & 0 & 0 & 25 & 0 & 0 \\
\hline 17.8 & 0 & 10 & 18 & 4 & 1 \\
\hline $17 \mathrm{~A}$ & 15 & 0 & 0 & 0 & 0 \\
\hline $17 \mathrm{~B}$ & 46 & 0 & 0 & 0 & 0 \\
\hline $17 \mathrm{D}$ & 1 & 0 & 0 & 0 & 0 \\
\hline 19 & 0 & 1 & 0 & 0 & 0 \\
\hline 19.4 & 0 & 27 & 0 & 0 & 0 \\
\hline $19 \mathrm{~A}$ & 14 & 0 & 0 & 0 & 0 \\
\hline $19 D$ & 0 & 0 & 8 & 0 & 0 \\
\hline $19 \mathrm{G}$ & 0 & 0 & 4 & 0 & 0 \\
\hline $1 \mathrm{~A}$ & 16 & 0 & 0 & 0 & 0 \\
\hline 2.1 & 0 & 0 & 10 & 5 & 0 \\
\hline 21.4 & 0 & 10 & 7 & 0 & 0 \\
\hline 22.1 & 0 & 0 & 4 & 0 & 0 \\
\hline 24.1 & 0 & 0 & 4 & 0 & 0 \\
\hline $24.1 \mathrm{~A}$ & 0 & 0 & 0 & 7 & 0 \\
\hline $24.1 B$ & 0 & 0 & 0 & 6 & 7 \\
\hline 24.4 & 0 & 0 & 9 & 0 & 0 \\
\hline 24.5 & 0 & 0 & 0 & 50 & 10 \\
\hline 25 & 1 & 0 & 0 & 0 & 1 \\
\hline 28 & 1 & 2 & 0 & 0 & 0 \\
\hline 28.2 & 0 & 0 & 22 & 0 & 0 \\
\hline 28.3 & 0 & 0 & 20 & 8 & 0 \\
\hline 28.4 & 0 & 2 & 0 & 0 & 2 \\
\hline 28.5 & 0 & 22 & 10 & 0 & 0 \\
\hline 28.7 & 0 & 0 & 0 & 15 & 0 \\
\hline $28 \mathrm{~A}$ & 23 & 0 & 0 & 0 & 0 \\
\hline 29 & 1 & 0 & 0 & 0 & 0 \\
\hline
\end{tabular}

\begin{tabular}{|c|c|c|c|c|c|}
\hline \multicolumn{6}{|c|}{$\begin{array}{c}\text { Mendota Wildlife Area } \\
\text { Year }\end{array}$} \\
\hline Site & 1987 & 1988 & 1989 & 1990 & 1991 \\
\hline 29.6 & 0 & 0 & 13 & 0 & 0 \\
\hline $29 A$ & 10 & 0 & 0 & 0 & 0 \\
\hline $29 B$ & 1 & 0 & 0 & 0 & 0 \\
\hline $2 \mathrm{~A}$ & 2 & 0 & 0 & 0 & 0 \\
\hline 3 & 0 & 1 & 0 & 0 & 0 \\
\hline 3.4 & 0 & 4 & 5 & 0 & 0 \\
\hline 30 & 0 & 1 & 0 & 0 & 0 \\
\hline 30.1 & 0 & 13 & 11 & 0 & 0 \\
\hline 30.3 & 0 & 0 & 13 & 0 & 0 \\
\hline $30 \mathrm{~A}$ & 9 & 0 & 0 & 0 & 0 \\
\hline 31 & 1 & 0 & 0 & 0 & 0 \\
\hline $31 \mathrm{~A}$ & 6 & 0 & 0 & 0 & 0 \\
\hline 33.1 & 0 & 1 & 0 & 0 & 0 \\
\hline $33 \mathrm{~A}$ & 15 & 0 & 6 & 0 & 0 \\
\hline $33 B$ & 1 & 0 & 0 & 0 & 0 \\
\hline 34.3 & 0 & 0 & 14 & 0 & 0 \\
\hline 34.9 & 0 & 0 & 0 & 0 & 1 \\
\hline $34 \mathrm{~A}$ & 5 & 0 & 0 & 0 & 0 \\
\hline 4.7 & 0 & 0 & 1 & 0 & 0 \\
\hline 45 & 11 & 0 & 0 & 0 & 0 \\
\hline $45.2 \mathrm{~A}$ & 0 & 0 & 0 & 0 & 2 \\
\hline $46 \mathrm{~A}$ & 9 & 0 & 0 & 0 & 0 \\
\hline $46 \mathrm{~B}$ & 15 & 0 & 0 & 0 & 0 \\
\hline $46 C$ & 13 & 0 & 0 & 0 & 0 \\
\hline $4 \mathrm{~A}$ & 1 & 0 & 0 & 0 & 0 \\
\hline 8.2 & 0 & 0 & 3 & 1 & 0 \\
\hline 8.3 & 0 & 0 & 25 & 32 & 8 \\
\hline $8 \mathrm{~A}$ & 5 & 0 & 0 & 0 & 0 \\
\hline 9 & 0 & 2 & 0 & 0 & 0 \\
\hline 9.2 & 0 & 3 & 5 & 0 & 0 \\
\hline HQ & 1 & 0 & 0 & 0 & 0 \\
\hline Incidental & 14 & 1 & 19 & 5 & 0 \\
\hline \multicolumn{6}{|c|}{ Fresno Slough } \\
\hline Islanc & 16 & 0 & 61 & 0 & 0 \\
\hline PRA & 7 & 0 & 0 & 0 & 0 \\
\hline $\mathrm{PRB}$ & 40 & 0 & 0 & 0 & 0 \\
\hline $\mathrm{PRC}$ & 68 & 0 & 0 & 0 & 0 \\
\hline PRD & 34 & 0 & 0 & 0 & 0 \\
\hline PRE & 25 & 0 & 0 & 0 & 0 \\
\hline T47 & 0 & 0 & 11 & 22 & 7 \\
\hline T50 & 0 & 0 & 0 & 0 & 4 \\
\hline T50A & 0 & 0 & 0 & 0 & 15 \\
\hline T50B & 0 & 0 & 0 & 0 & 20 \\
\hline $\mathrm{T} 50 \mathrm{C}$ & 0 & 0 & 0 & 0 & 11 \\
\hline
\end{tabular}




\begin{tabular}{|c|c|c|c|}
\hline Mountain M & $\begin{array}{l}\text { Meadows } \\
\text { Year }\end{array}$ & Rese & ervoi \\
\hline Site & 1997 & 1999 & 2001 \\
\hline Mid & 2 & 0 & \\
\hline MMB & 1 & 0 & \\
\hline MMC & 1 & 0 & \\
\hline North & 2 & 0 & \\
\hline WL-Mid & 0 & 2 & \\
\hline WL-North & 0 & 1 & \\
\hline WL-South & 0 & 1 & \\
\hline
\end{tabular}

\section{Napa-Sonoma Marsh

$$
\text { Year }
$$ \\ Site 1994

110

Palm Tract

\begin{tabular}{lrrr} 
Site & $\begin{array}{r}\text { Year } \\
\mathbf{1 9 9 6}\end{array}$ & $\mathbf{1 9 9 7}$ & $\mathbf{1 9 9 8}$ \\
\hline $1-1$ & 1 & 0 & 0 \\
$1-2$ & 3 & 2 & 0 \\
$1-3$ & 3 & 1 & 1 \\
$1-4$ & 0 & 1 & 1 \\
$1-6$ & 4 & 5 & 0 \\
$2-1$ & 0 & 0 & 1 \\
$2-2$ & 3 & 1 & 0 \\
$2-3$ & 2 & 4 & 3 \\
$2-4$ & 1 & 3 & 2 \\
$2-5$ & 1 & 1 & 3 \\
$3-1$ & 0 & 2 & 2 \\
$3-2$ & 4 & 1 & 1 \\
$3-3$ & 0 & 1 & 0 \\
L2 & 0 & 0 & 10 \\
L3 & 0 & 0 & 1 \\
Levee & 4 & 0 & 0 \\
Levee 2 & 0 & 9 & 0 \\
Levee 3 & 0 & 2 & 0 \\
North Field & 0 & 2 & 0 \\
Season Wetland & 1 & 0 & 0 \\
SW3 & 0 & 0 & 1
\end{tabular}

\begin{tabular}{cr} 
Riley & $\begin{array}{r}\text { Farms } \\
\text { Year }\end{array}$ \\
Site & $\mathbf{1 9 8 6}$ \\
\hline Riley & 25
\end{tabular}


Sacramento National Wildlife Refuge

Year

$\begin{array}{llllll}\text { Site } & 1986 & 1987 & 1988 & 1990 & 1991\end{array}$

$\begin{array}{llllll}1 & 0 & 0 & 7 & 0 & 0\end{array}$

$\begin{array}{llllll}14 & 0 & 0 & 2 & 0 & 0\end{array}$

IS $\quad 0 \quad 0 \quad 001$

$\begin{array}{lrrrrr}151 & 0 & 0 & 0 & 0 & 3\end{array}$

$\begin{array}{llllll}25 & 63 & 81 & 35 & 0 & 0\end{array}$

$\begin{array}{llllll}31 R & 0 & 0 & 1 & 0 & 0 \\ 36 R & 0 & 0 & 1 & 0 & 0\end{array}$

$\begin{array}{llllll}41 & 0 & 0 & 2 & 0 & 0 \\ 42 & 0 & 2 & 2 & 0 & 0\end{array}$

$43 \quad 43 \quad 60 \quad 40$

$\begin{array}{rrrrr}51 & 0 & 0 & 2 & 0\end{array}$

$\begin{array}{lllll}57 & 0 & 1 & 0 & 0 \\ 58 & 0 & 2 & 1 & 0\end{array}$

$\begin{array}{lllll}82 & 0 & 1 & 2 & 0\end{array}$

A $1-0=0$

$\begin{array}{rrrrr}\text { Bpa } & 0 & 0 & 0 & 0\end{array}$

$\begin{array}{rrrrrr}\mathrm{BPb} & 0 & 0 & 0 & 0 & 1\end{array}$

$\begin{array}{llllll}\mathrm{BPC} & 0 & 0 & 0 & 0 & 10\end{array}$

$\begin{array}{llllll}\mathrm{C} 1 & 0 & 0 & 0 & 0 & 43\end{array}$

$\begin{array}{llllll}\mathrm{C} 2 \mathrm{~A} & 0 & 0 & 0 & 0 & 12\end{array}$

$\begin{array}{llllll}\mathrm{C} 3 & 0 & 0 & 0 & 0 & 24\end{array}$

$\begin{array}{lllllr}\text { D1 } & 0 & 0 & 0 & 2 & 0\end{array}$

$\begin{array}{llllll}\text { D2 } & 0 & 0 & 0 & 0 & 7\end{array}$

$\begin{array}{rrrrrr}\text { F1 } & 0 & 0 & 0 & 2 & 3\end{array}$

$\begin{array}{llllll}\mathrm{F} 2 & 0 & 0 & 0 & 31 & 5\end{array}$

$\begin{array}{rrrrrr}\text { FS1 } & 0 & 0 & 0 & 0 & 37 \\ \text { G1 } & 0 & 0 & 0 & 7 & 0\end{array}$

GR1

H1 0

H2 $\quad 0$

H3 $\mathrm{O}$

INC 2

J1

J2

$\mathrm{J}$

JM2
Sacramento National Wildlife Refuge

Year

Site $\quad \begin{array}{lllll}1986 & 1987 & 1988 & 1990 & 1991\end{array}$

$\begin{array}{rrrrrr}\text { JS1 } & 0 & 0 & 0 & 0 & 1 \\ \text { JS2 } & 0 & 0 & 0 & 0 & 21\end{array}$

$\begin{array}{llllll}\text { JS3 } & 0 & 0 & 0 & 0 & 27\end{array}$

$\begin{array}{llllll}\text { JUS } & 0 & 2 & 0 & 0 & 0\end{array}$

$\begin{array}{llllll}\text { L1 } & 0 & 0 & 0 & 89 & 0\end{array}$

$\begin{array}{lllllr}\text { L2 } & 0 & 0 & 0 & 58 & 0\end{array}$

$\begin{array}{llllll}\text { L6 } & 0 & 0 & 0 & 0 & 25\end{array}$

$\begin{array}{llllll}\text { L7 } & 0 & 0 & 0 & 0 & 41\end{array}$

$\begin{array}{llllll}\text { M2 } & 0 & 0 & 0 & 33 & 190\end{array}$

$\begin{array}{llllll}\text { M3 } & 0 & 0 & 0 & 6 & 3\end{array}$

$\begin{array}{llllll}\text { M4 } & 0 & 0 & 0 & 1 & 0\end{array}$

$\begin{array}{llllll}\text { ME1 } & 0 & 0 & 0 & 0 & 24\end{array}$

$\begin{array}{llllll}\text { ME2 } & 0 & 0 & 0 & 0 & 4\end{array}$

$\begin{array}{llllll}M E 3 & 0 & 0 & 0 & 0 & 20\end{array}$

$\begin{array}{llllll}\text { P1 } & 0 & 0 & 0 & 2 & 0 \\ \text { P2 } & 0 & 0 & 0 & 1 & 0\end{array}$

$\begin{array}{llllll}P 2 & 0 & 0 & 0 & 1 & 0 \\ P D 1 & 0 & 0 & 0 & 0 & 80\end{array}$

$\begin{array}{llllll}\text { RM1 } & 0 & 0 & 0 & 0\end{array}$

$\begin{array}{llllll}\text { S1 } & 0 & 0 & 0 & 4 & 10\end{array}$

$\begin{array}{llllll}S 4 & 0 & 0 & 0 & 8 & 0\end{array}$

$\begin{array}{llllll}\text { SB1 } & 0 & 0 & 0 & 0 & 4\end{array}$

$\begin{array}{llllll}\mathrm{SB} 2 & 0 & 0 & 0 & 0 & 2\end{array}$

$\begin{array}{llllll}\text { SB3 } & 0 & 0 & 0 & 0 & 2\end{array}$

$\begin{array}{llllll}\mathrm{T} 16 & 0 & 1 & 0 & 0 & 0\end{array}$

$\begin{array}{llllll}T 1 A & 0 & 0 & 0 & 0 & 21\end{array}$

$\begin{array}{llllll}\text { T1B } & 0 & 0 & 0 & 0 & 14\end{array}$

$\begin{array}{llllll}\mathrm{T} 1 \mathrm{C} & 0 & 0 & 0 & 0 & 15\end{array}$

$\begin{array}{llllll}\text { T2A } & 0 & 0 & 0 & 0 & 11\end{array}$

T2C $00 \begin{array}{lllll} & 0 & 0 & 0 & 1\end{array}$

$\begin{array}{llllll}T 3 & 0 & 0 & 0 & 0 & 1\end{array}$

$\begin{array}{llllll}\mathrm{T} 4 \mathrm{~b} & 0 & 0 & 0 & 0 & 28\end{array}$

$\begin{array}{llllll}\text { TG } & 0 & 0 & 13 & 0 & 0\end{array}$

$\begin{array}{llllll}T V 1 & 0 & 0 & 0 & 0 & 21\end{array}$

$\begin{array}{llllll}\text { TV2 } & 0 & 0 & 0 & 0 & 19\end{array}$

$\begin{array}{llllll}\text { TV3 } & 0 & 0 & 0 & 0 & 14\end{array}$

$\begin{array}{llllll}Y 06 & 0 & 0 & 1 & 0 & 0\end{array}$

$\begin{array}{llllll}\text { Y07 } & 0 & 0 & 2 & 0 & 0 \\ \text { zum } & 0 & 0 & 6 & 0 & 0\end{array}$ 


\begin{tabular}{|c|c|c|}
\hline ite & $\begin{array}{l}\text { Year } \\
1992\end{array}$ & 1993 \\
\hline 1 & 1 & $\overline{0}$ \\
\hline 2 & 10 & 0 \\
\hline 3 & 12 & 0 \\
\hline N21 & 0 & 1 \\
\hline W21 & 0 & 1 \\
\hline
\end{tabular}

\begin{tabular}{|c|c|c|c|c|c|c|}
\hline \multicolumn{2}{|c|}{$\begin{array}{r}\text { Thermalito } \\
\text { Year }\end{array}$} & \multicolumn{2}{|c|}{ Afterbay } & \multirow[b]{2}{*}{1990} & \multirow[b]{2}{*}{1993} & \multirow[b]{2}{*}{1994} \\
\hline Site & 1987 & 1988 & 1989 & & & \\
\hline $1 \mathrm{E}$ & 0 & 0 & 3 & 0 & 0 & $\overline{0}$ \\
\hline $1 \mathrm{E} 1$ & 0 & 0 & 0 & 2 & 2 & 18 \\
\hline 1E10 & 0 & 0 & 0 & 0 & 0 & 1 \\
\hline $1 \mathrm{E} 1 \mathrm{~A}$ & 0 & 0 & 0 & 12 & 6 & 8 \\
\hline $1 E 1 B$ & 0 & 0 & 0 & 2 & 4 & 19 \\
\hline $1 \mathrm{E} 2$ & 0 & 0 & 2 & 3 & 0 & 6 \\
\hline $1 \mathrm{E} 2 \mathrm{~A}$ & 0 & 0 & 0 & 0 & 4 & 2 \\
\hline $1 \mathrm{E} 2 \mathrm{~B}$ & 0 & 0 & 0 & 0 & 1 & 2 \\
\hline $1 \mathrm{E} 3$ & 0 & 0 & 1 & 0 & 1 & 0 \\
\hline $1 \mathrm{E} 4$ & 0 & 0 & 1 & 1 & 0 & 4 \\
\hline $1 \mathrm{E} 4 \mathrm{~A}$ & 0 & 0 & 0 & 0 & 1 & 3 \\
\hline $1 \mathrm{E} 5$ & 0 & 0 & 10 & 5 & 0 & 0 \\
\hline $1 \mathrm{E} 5 \mathrm{~A}$ & 0 & 0 & 0 & 5 & 1 & 8 \\
\hline $1 E 5 B$ & 0 & 0 & 0 & 0 & 1 & 1 \\
\hline $1 \mathrm{E} 5 \mathrm{C}$ & 0 & 0 & 0 & 0 & 1 & 1 \\
\hline $1 \mathrm{E} 6$ & 0 & 0 & 0 & 0 & 8 & 28 \\
\hline $1 \mathrm{E} 7$ & 0 & 0 & 0 & 0 & 4 & 29 \\
\hline $1 \mathrm{E} 8$ & 0 & 0 & 0 & 0 & 11 & 47 \\
\hline $1 \mathrm{~N} 1 \mathrm{a}$ & 0 & 0 & 0 & 0 & 1 & 5 \\
\hline $1 \mathrm{~N} 2$ & 0 & 0 & 0 & 13 & 0 & 0 \\
\hline $1 \mathrm{~N} 2 \mathrm{~A}$ & 0 & 0 & 0 & 0 & 2 & 1 \\
\hline $1 \mathrm{~N} 2 \mathrm{~B}$ & 0 & 0 & 0 & 0 & 2 & 2 \\
\hline $1 \mathrm{~N} 3$ & 0 & 0 & 0 & 3 & 2 & 0 \\
\hline $1 \mathrm{~N} 4$ & 0 & 0 & 0 & 0 & 1 & 0 \\
\hline $1 \mathrm{~N} 5$ & 0 & 0 & 0 & 0 & 7 & 6 \\
\hline $1 \mathrm{~N} 6$ & 0 & 0 & 0 & 0 & 5 & 2 \\
\hline $1 \mathrm{~N} 7$ & 0 & 0 & 0 & 0 & 0 & 1 \\
\hline $1 \mathrm{~S} 2$ & 0 & 0 & 0 & 0 & 3 & 0 \\
\hline $4 E$ & 19 & 1 & 4 & 0 & 0 & 0 \\
\hline $4 \mathrm{~N}$ & 18 & 6 & 6 & 0 & 0 & 0 \\
\hline $4 \mathrm{~N} 1$ & 0 & 1 & 0 & 0 & 0 & 0 \\
\hline $4 \mathrm{~N} 1 \mathrm{~A}$ & 0 & 0 & 0 & 1 & 0 & 0 \\
\hline $4 \mathrm{~N} 2$ & 0 & 3 & 2 & 0 & 0 & 0 \\
\hline $4 \mathrm{~N} 3$ & 0 & 0 & 2 & 0 & 0 & 0 \\
\hline $4 N 4$ & 0 & 0 & 3 & 0 & 0 & 0 \\
\hline 4SW & 7 & 0 & 0 & 0 & 0 & 0 \\
\hline $4 W$ & 6 & 0 & 1 & 1 & 0 & 0 \\
\hline $5 \mathrm{~N}$ & 6 & 0 & 0 & 0 & 0 & 0 \\
\hline $5 \mathrm{~N} 1$ & 0 & 4 & 0 & 0 & 0 & 0 \\
\hline B1 & 0 & 0 & 0 & 0 & 0 & 5 \\
\hline B2 & 0 & 0 & 0 & 0 & 0 & 2 \\
\hline $\mathrm{C} 1$ & 0 & 0 & 0 & 0 & 0 & 1 \\
\hline $\mathrm{C} 2$ & 0 & 0 & 0 & 0 & 0 & 2 \\
\hline E1 & 0 & 8 & 0 & 0 & 0 & 0 \\
\hline TP2 & 0 & 2 & 0 & 0 & 0 & 0 \\
\hline
\end{tabular}




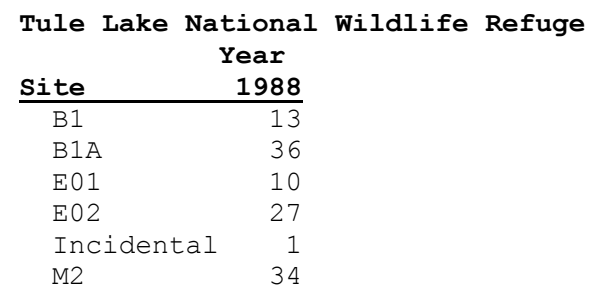

\begin{tabular}{|c|c|}
\hline Winte & $\begin{array}{l}\text { ers } \\
\text { Year }\end{array}$ \\
\hline Site & 1988 \\
\hline 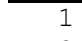 & \\
\hline 3 & \\
\hline
\end{tabular}

\begin{tabular}{lrrr} 
Yolo Bypass & \multicolumn{3}{c}{$\begin{array}{r}\text { Year } \\
\text { Site }\end{array}$} \\
\hline 1986 & $\mathbf{1 9 8 8}$ & $\mathbf{2 0 0 8}$ \\
\hline C2 & 0 & 0 & 8 \\
IN1 & 0 & 1 & 0 \\
Parker 1 & 0 & 0 & 3 \\
Senator Outing 1 & 11 & 0 & 0 \\
Senator Outing 2 & 11 & 0 & 0 \\
YO1 & 0 & 8 & 0 \\
YO2 & 0 & 3 & 0 \\
YO5 & 0 & 16 & 0 \\
YO6 & 0 & 8 & 0 \\
YO7 & 0 & 17 & 0 \\
YolO Flyway & 5 & 0 & 0
\end{tabular}


Publishing support provided by the U.S. Geological Survey

Science Publishing Network, Tacoma Publishing Service Center

For more information concerning the research in this report, contact the Director, Western Ecological Research Center

U.S. Geological Survey

3020 State University Drive East

Sacramento, California 95819

http://werc.usgs.gov/ 


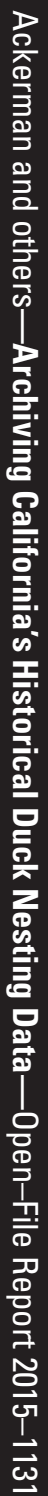

